\title{
The Sommerfeld Half-plane Problem Revisited, IV: Variations on a Theme of Carlson and Heins
}

\author{
A. E. Heins* and J. M. Pond \\ University of Michigan, Ann Arbor, MI 48109, U.S.A. \\ Communicated by E. Meister
}

\begin{abstract}
A plane wave is incident upon an infinite set of equally spaced, semi-infinite parallel and staggered plates. The boundary conditions on the plates alternate between the Dirichlet and Neumann ones. This problem is formulated as a pair of coupled Wiener-Hopf integral equations and solved by a method proposed by A. E. Heins in 1950. For the case of specular reflection, that is, a single reflected plane wave, the magnitudes of the reflection coefficient and the transmission coefficients are determined.
\end{abstract}

\section{Introduction}

We shall discuss here the reflection and transmission properties of a plane wave which is incident upon an infinite stack of staggered and equally spaced parallel plates. Here we shall assume that the boundary conditions on the plates alternate between the Dirichlet and Neumann ones. Carlson and Heins ${ }^{1}$ originally considered such a stack of plates which obeyed either Dirichlet or Neumann boundary conditions and were led to solve a single integral equation of the Wiener-Hopf type. The problem we propose here now leads to a pair of such equations, and a method proposed by Heins $^{4}$ in 1948 will provide the solution. (See also the paper of Daniele. ${ }^{2}$ This author stated in correspondence dated 7 September 1981, to A. E. Heins that his work is based on Reference 4.) We shall formulate the present problem with the aid of a periodic Green's function constructed by Heins ${ }^{3}$ in 1957 and thereby be spared the use of the artifice that the wave number $k$ have a positive imaginary part. The magnitudes of the amplitudes of the specularly reflected wave and the transmission coefficients for the dominant propagating wave in two consecutive ducts will be given. (There are two distinct transmission coefficients.) We shall find that magnitude of the specularly reflected wave can be calculated in a particularly simple manner by a method introduced in Reference 3. In fact, this reflection coefficient has the same magnitude as the one which was derived in Part I of the Carlson and Heins paper. ${ }^{1}$

A special case of this problem has recently been discussed by Hurd and Lüneburg. ${ }^{6}$ There is no stagger in their configuration and they did not find the explicit magnitudes

* Department of Mathematics

†Department of Electrical Engineering and Computer Science 
of the specularly reflected or the transmitted waves. Since they had not taken into account all of the properties of the periodic Green's function, they found it necessary to introduce a positive imaginary part for the wave number in order to carry out their analysis.

The method which we employ to solve this problem depends on our ability to 'factor' the matrix of the Fourier transforms of the kernels in the system of Wiener-Hopf integral equations which we shall derive. As we shall see in Section 4, it is possible to 'factor' the matrix in the present case, in the same sense that Wiener and Hopf did it for the scalar case. That it is possible, in this instance, is due to the fact that there is basically only one independent function to factor - a situation which does not occur very often. The method relies heavily on two basic ideas: (a) Sylvester's theorem ${ }^{5}$ regarding the function of a matrix and (b) analytic continuation.

\section{Formulation of the problem}

We recall that if a monochromatic plane wave is incident upon an infinite stack of semi-infinite, equally spaced, staggered parallel plates with a Dirichlet boundary condition on the plates $y=2 n b, n=0, \pm 1, \pm 2, \ldots$ and a Neumann boundary condition on the plates $y=(2 n+1) b, n=0, \pm 1, \pm 2, \ldots$ (see Fig. 1) that

$$
\begin{aligned}
\phi(x, y)= & -\int_{0}^{\infty} G\left(x, y ; x^{\prime}, 0\right) \frac{\partial \phi\left(x^{\prime}, 0+\right)}{\partial y^{\prime}} \mathrm{d} x^{\prime}+\int_{a}^{\infty}[\phi] \frac{\partial G\left(x, y ; x^{\prime}, b\right)}{\partial y^{\prime}} \mathrm{d} x^{\prime} \\
& +\int_{2 a}^{\infty} G\left(x, y ; x^{\prime}, 2 b\right) \frac{\partial \phi\left(x^{\prime}, 2 b-\right)}{\partial y^{\prime}} \mathrm{d} x^{\prime}+\exp \left[\mathrm{i}\left(x k_{x}+y k_{y}\right)\right] \\
& +R \exp \left[\mathrm{i}\left(x k_{x}^{*}+y k_{y}^{*}\right)\right]
\end{aligned}
$$

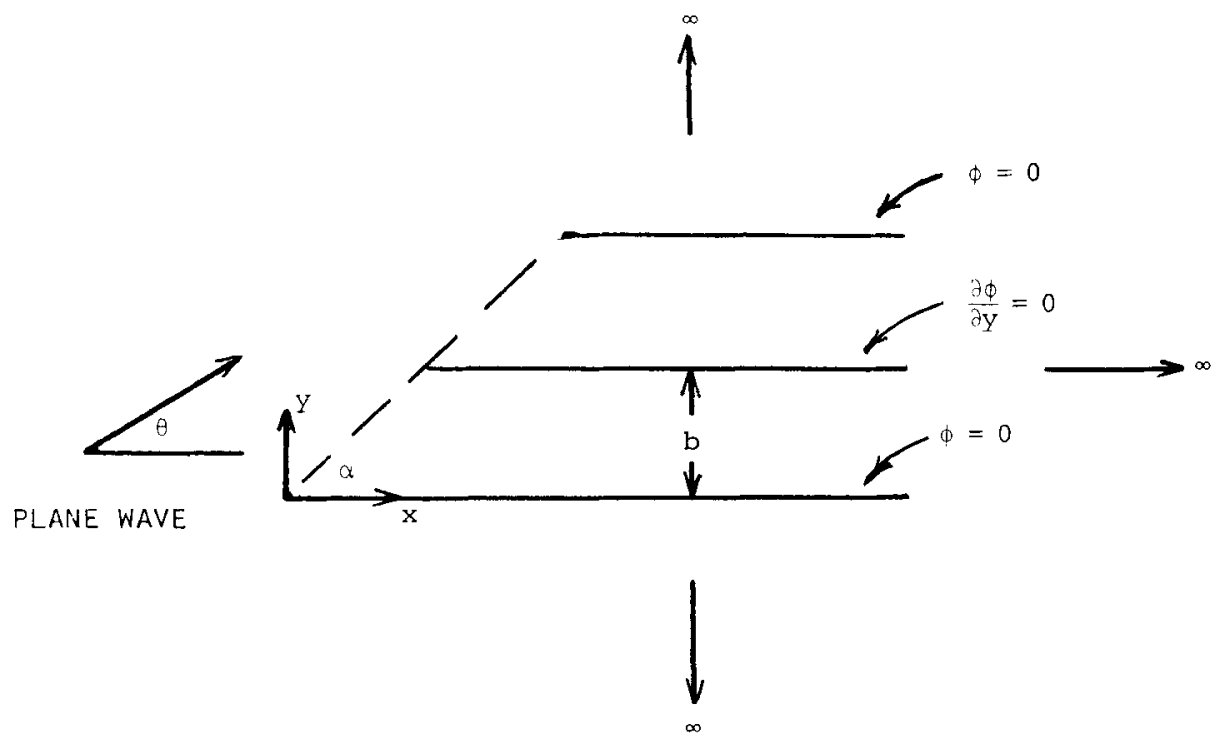

Fig. 1 . 
is a representation of a solution of the equation

$$
\phi_{x x}+\phi_{y y}+k^{2} \phi=0
$$

The notation $\phi(x, 0+)$ implies that $\lim _{y \rightarrow 0+} \phi(x, y)=\phi(x, 0+)$ and $\partial \phi(x, 0+) / \partial y^{\prime} \mathrm{im}-$ plies that

$$
\lim _{y^{\prime} \rightarrow 0^{+}} \frac{\partial \phi\left(x^{\prime}, y^{\prime}\right)}{\partial y^{\prime}}=\frac{\partial \phi\left(x^{\prime}, 0+\right)}{\partial y^{\prime}} .
$$

Similar interpretations are available for $\phi\left(x^{\prime}, 2 b-\right)$, etc. $(2.1)$ incorporates the Sommerfeld radiation condition for plane waves, the incident wave $\exp \left[\mathrm{i}\left(x k_{x}+y k_{y}\right)\right]$, the specularly reflected wave $\exp \left[\mathrm{i}\left(x k_{x}^{*}+y k_{y}^{*}\right)\right]$ and the periodic nature of the structure. Although we have assumed that there is only the specularly reflected wave, it is possible to include diffracted plane waves. ${ }^{3}$ The Green's function $G\left(x, y ; x^{\prime}, y^{\prime}\right)$ is a solution of the equation

$$
G_{x x}+G_{y y}+k^{2} G=0
$$

save at the point $P(x, y)=P^{\prime}\left(x^{\prime}, y^{\prime}\right)$ in the strip $-\infty<x, x^{\prime}<+\infty, 0 \leq y, y^{\prime}<2 b$ where it has a logarithmic singularity. It is subject to the following periodic boundary conditions which will subsequently simplify our work:

$$
G\left(x, y ; x^{\prime}+2 a, 2 b\right) \exp \left[2 \mathrm{i}\left(a k_{x}+b k_{y}\right)\right]=G\left(x, y ; x^{\prime}, 0\right),
$$

and there is a similar condition on the $y^{\prime}$ derivative. The construction of this Green's function is to be found in Reference 3. The symbol [ $\phi]$ denotes the discontinuity of $\phi$ on the plate $y=b . k_{x}$ and $k_{y}$ are the $x$ and $y$ components of the propagation normal of the incident plane wave, whereas $k_{x}^{*}$ and $k_{y}^{*}$ are the corresponding components for the propagation normal of the specularly reflected wave.

In the view of the fact, in the present case, that $\partial \phi(x, 2 b-) / \partial y=\exp \left[2 \mathrm{i}\left(a k_{x}\right.\right.$ $\left.\left.+b k_{y}\right)\right] \partial \phi(x, 0-) / \partial y$, the third integral in (2.1) may be rewritten as

$$
\begin{aligned}
\int_{2 a}^{\infty} G\left(x, y ; x^{\prime}, 2 b\right) \frac{\partial \phi\left(x^{\prime}, 2 b-\right)}{\partial y} \mathrm{~d} x^{\prime} & =\int_{0}^{\infty} G(x, y ; 2 \mathrm{a}+t, 2 b) \frac{\partial \phi(2 a+t, 2 b-)}{\partial y^{\prime}} \mathrm{d} t \\
& =\int_{0}^{\infty} G(x, y ; t, 0) \frac{\partial \phi(t, 0-)}{\partial y^{\prime}} \mathrm{d} t .
\end{aligned}
$$

Hence (2.1) becomes

$$
\begin{aligned}
\phi(x, y)= & -\int_{0}^{\infty} G\left(x, y ; x^{\prime}, 0\right)\left[\frac{\partial \phi}{\partial y^{\prime}}\right] \mathrm{d} x^{\prime}+\int_{a}^{\infty}[\phi] \frac{\partial G\left(x, y ; x^{\prime}, b\right)}{\partial y^{\prime}} \mathrm{d} x^{\prime} \\
& +\exp \left[\mathrm{i}\left(x k_{x}+y k_{y}\right)\right]+R \exp \left[\mathrm{i}\left(x k_{x}^{*}+y k_{y}^{*}\right]\right),
\end{aligned}
$$

where now $\left[\partial \phi / \partial y^{\prime}\right]$ denotes the discontinuity of the $y^{\prime}$ derivative of $\phi$ on the sheet $y^{\prime}$ $=0, x^{\prime}>0 . R$ is the reflection coefficient of the specularly reflected wave which we shall ultimately compute. 
The Green's function has the following integral representation:

$$
\begin{aligned}
& G\left(x, y ; x^{\prime}, y^{\prime}\right)=\frac{1}{2 \pi} \int_{\mathscr{Z}} \frac{\exp [2 \mathrm{i} \lambda(w)] \sin \left[\left(y^{\prime}-y\right) \sigma(w)\right]-\sin \left[\left(y^{\prime}-y+2 b\right) \sigma(w)\right]}{2 \sigma(w)[\cos 2 \hat{\lambda}(w)-\cos 2 b \sigma(w)]} \\
& \quad \times \exp \left[\mathrm{i} w\left(x-x^{\prime}\right)\right] \mathrm{d} w, \quad y>y^{\prime} \\
& =\frac{1}{2 \pi} \int_{\mathscr{Z}}^{\infty} \frac{\sin \left[\left(y^{\prime}-y-2 b\right) \sigma(w)\right]-\exp [-2 \mathrm{i} \lambda(w)] \sin \left[\left(y^{\prime}-y\right) \sigma(w)\right]}{2 \sigma(w)[\cos 2 \hat{\lambda}(w)-\cos 2 h \sigma(w)]} \\
& \quad \times \exp \left[\mathrm{i} w\left(x-x^{\prime}\right)\right] \mathrm{d} w, \quad y<y^{\prime}
\end{aligned}
$$

where $\lambda(w)=a k_{x}+b k_{y}-a w$. Now since $\tan \alpha=b / a$ where $\alpha$ is the angle of stagger, we shall express $k_{x}=k \cos \theta$ and $k_{y}=k \sin \theta$ (where $\theta$ is the angle which the propagation normal of the incident plane wave makes with respect to the positive $x$-axis) and write $\lambda=c k \cos (\theta-\alpha)-a w$ where $c=\sqrt{ }\left(a^{2}+b^{2}\right)$. In view of the fact that the integrands in (2.4) are meromorphic functions of $w$, we shall take $\sigma(w)=\sqrt{ }\left(k^{2}-w^{2}\right)$ to be real and positive for $w=0 . \mathscr{L}$ is a path of integration parallel to the real axis of the $w$-plane and it is drawn in the strip $-\varepsilon<\operatorname{Im} w<0$. Here $\varepsilon$ is the imaginary part of that complex root of $\cos 2 \lambda-\cos 2 b \sigma=0$ with the smallest imaginary part. That is, ${ }^{3} \varepsilon$ is the smaller of $b\left\{[\pi-k c \cos (\theta-\alpha)]^{2}-c^{2} k^{2}\right\} / c^{2}$ and $b\left\{[\pi+k c \cos (\theta-\alpha)]^{2}-c^{2} k^{2}\right\} / c^{2}$, both of which are real since we are only examining the case of specular reflection.

Upon applying the boundary conditions on the plates $y=0, x>0$ and $y=b, x>a$, we obtain a system of two integral equations of the Wiener-Hopf type. That is

$$
\begin{aligned}
& -\int_{0}^{\infty} G\left(x, 0 ; x^{\prime}, 0\right)\left[\frac{\partial \phi}{\partial y^{\prime}}\right] \mathrm{d} x^{\prime}+\int_{a}^{\infty}[\phi] \frac{\partial G\left(x, 0 ; x^{\prime}, b\right)}{\partial y^{\prime}} \mathrm{d} x^{\prime} \\
& +\exp \left[\mathrm{i} x k_{x}\right]+R \exp \left[\mathrm{i} x k_{x}^{*}\right]=0, \quad x>0, \quad y=0
\end{aligned}
$$

and

$$
\begin{aligned}
& -\frac{\partial}{\partial y} \int_{0}^{\infty} G\left(x, y ; x^{\prime}, 0\right)\left[\frac{\partial \phi}{\partial y^{\prime}}\right] \mathrm{d} x^{\prime}+\frac{\partial}{\partial y} \int_{a}^{\infty}[\phi] \frac{\partial G\left(x, y ; x^{\prime}, b\right)}{\partial y^{\prime}} \mathrm{d} x^{\prime} \\
& +i k_{y} \exp \left[\mathrm{i}\left(x k_{x}+b k_{y}\right)\right]+\mathrm{i} k_{y}^{*} R \exp \left[\mathrm{i}\left(x k_{x}^{*}+b k_{y}^{*}\right)\right]=0, \quad x>a, \quad y=b .
\end{aligned}
$$

Following Wiener and Hopf, we rewrite this system, valid for $-\infty<x<\infty$ as follows. We put

$$
\begin{array}{rlrl}
{\left[\frac{\partial \phi}{\partial y^{\prime}}\right]} & \equiv f_{1}(x), & & x>0, \\
& \equiv 0, & & x<0, \\
{[\phi]} & \equiv f_{2}(x), & & x>a, \\
& \equiv 0, & & x<a, \\
\frac{\partial \phi(x, b)}{\partial y} & \equiv F_{1}(x), & x<a, \\
& \equiv 0, & & x>a, \\
\phi(x, 0) & \equiv F_{2}(x), & x<0, \\
& \equiv 0, & & x>0,
\end{array}
$$




$$
\begin{aligned}
\phi_{1}^{*}(x) & \equiv \exp \left(\mathrm{i} x k_{x}\right)+R \exp \left(\mathrm{i} x k_{x}^{*}\right), & & x>0, \\
& \equiv 0, & x<0, & \\
\phi_{2}^{*}(x) & \equiv \mathrm{i} k_{y} \exp \left[\mathrm{i}\left(x k_{x}+b k_{y}\right)\right]+\mathrm{i} k_{y}^{*} R \exp \left[\mathrm{i}\left(x k_{x}^{*}+b k_{y}^{*}\right)\right], & & x>a, \\
& \equiv 0, & & x<a .
\end{aligned}
$$

Hence (2.5) and (2.6) may now be rewritten as

$$
F_{2}(x)=\phi_{1}^{*}(x)-\int_{-\infty}^{\infty} G\left(x, 0 ; x^{\prime}, 0\right) f_{1}\left(x^{\prime}\right) \mathrm{d} x^{\prime}+\int_{-\infty}^{\infty} f_{2}\left(x^{\prime}\right) \frac{\partial G\left(x, 0 ; x^{\prime}, b\right)}{\partial y^{\prime}} \mathrm{d} x^{\prime}
$$

and

$$
\begin{aligned}
F_{1}(x)= & \phi_{2}^{*}(x)-\frac{\partial}{\partial y} \int_{-\infty}^{\infty} G\left(x, y ; x^{\prime}, 0\right) f_{1}\left(x^{\prime}\right) \mathrm{d} x^{\prime} \\
& +\frac{\partial}{\partial y} \int_{-\infty}^{\infty} f_{2}\left(x^{\prime}\right) \frac{\partial G\left(x, y ; x^{\prime}, b\right)}{\partial y^{\prime}} \mathrm{d} x^{\prime}, \quad y=b-
\end{aligned}
$$

\section{The Fourier transform of the system (2.5)}

The determination of the Fourier transform of the system can be carried out without any difficulty. We know, for example, that $F_{1}(x)$ and $F_{2}(x)=O[\exp (\varepsilon x)], x \rightarrow$ $-\infty$ where $-\varepsilon<\operatorname{Im} w<0$, as we can conclude directly from the system (2.5). We also assume that $f_{1}(x)$ and $F_{2}(x)$ are integrable in the neighbourhood of the origin and that $f_{2}(x)$ and $F_{1}(x)$ are integrable in the neighbourhood of $x=a$. Since the parallel plate region can sustain only one propagating mode for the values of $c k$ which we consider here, we have that $f_{1}(x)$ and $f_{2}(x)=O(1), x \rightarrow \infty$. Hence the unilateral Fourier transforms of $F_{1}(x)$ and $F_{2}(x)$, that is

and

$$
\int_{-\infty}^{a} \exp [-\mathrm{i} w x] F_{1}(x) \mathrm{d} x=\hat{F}_{1}(w)
$$

$$
\int_{-\infty}^{0} \exp [-\mathrm{i} w x] F_{2}(x) \mathrm{d} x=\hat{F}_{2}(w)
$$

are analytic in the upper half-plane $\operatorname{Im} w>-\varepsilon$. Furthermore, the Fourier transforms of $f_{1}(x)$ and $f_{2}(x)$, that is

and

$$
\int_{0}^{\infty} \exp (-\mathrm{i} w x) f_{1}(x) \mathrm{d} x=\hat{f}_{1}(w)
$$

$$
\int_{a}^{\infty} \exp (-i w x) f_{2}(x) \mathrm{d} x=\hat{f}_{2}(w)
$$

are analytic in the lower half-plane $\operatorname{Im} w<0$. Notice that $\hat{F}_{1}(w)$ and $\hat{f}_{2}(w)$ possess exponential behaviour in their respective half-planes of analyticity, which we shall have to eliminate in order to apply the Liouville theorem.

We also require the Fourier transforms of $G(x, 0 ; 0,0) ; \partial G\left(x, 0 ; 0, y^{\prime}\right) / \partial y^{\prime}, y^{\prime}=b$; $\partial G(x, y ; 0,0) / \partial y, y=b$ and $\partial^{2} G\left(x, y ; 0, y^{\prime}\right) / \partial y \partial y^{\prime}, y=y^{\prime}=b$. From (2.4) it follows that 


$$
\begin{aligned}
& \int_{-\infty}^{\infty} G(x, 0 ; 0,0) \exp (-\mathrm{i} w x) \mathrm{d} x=-\frac{\sin 2 \sigma b}{2 \sigma[\cos 2 \lambda-\cos 2 \sigma b]} \\
& \left.\frac{\partial}{\partial y^{\prime}} \int_{-\infty}^{x} G\left(x, 0 ; 0, y^{\prime}\right)\right|_{y^{\prime}=b} \exp (-\mathrm{i} w x) \mathrm{d} x=\frac{\cos \sigma b[1-\exp (-2 \mathrm{i} \lambda)]}{2[\cos 2 \lambda-\cos 2 \sigma b]}, \\
& \left.\frac{\partial}{\partial y} \int_{-\infty}^{x} G(x, y ; 0,0)\right|_{y=b} \exp (-\mathrm{i} w x) \mathrm{d} x=\frac{\cos \sigma b[1-\exp (2 \mathrm{i} \lambda)]}{2[\cos 2 \lambda-\cos 2 \sigma b]}
\end{aligned}
$$

and

$$
\left.\frac{\partial^{2}}{\partial y \partial y^{\prime}} \int_{-x}^{x} G\left(x, y ; 0, y^{\prime}\right)\right|_{y=y^{\prime}=b} \exp (-\mathrm{i} w x) \mathrm{d} x=\frac{-\sigma \sin 2 \sigma b}{2[\cos 2 \lambda-\cos 2 \sigma b]}
$$

Here $\lambda=k \rho c-w a$ and $c \rho=a k_{x}+b k_{y}=k c \cos (\alpha-\theta)$. These transforms are analytic in the strip $-\varepsilon<\operatorname{Im} w<0$. The unilateral Fourier transforms of $\phi_{1}^{*}(x)$ and $\phi_{2}^{*}(x)$ are analytic in the lower half-plane $\operatorname{Im} w<0$. Hence we have in the strip $-\varepsilon<\operatorname{Im} w<0$

$$
\begin{aligned}
\hat{F}_{2}(w)= & \frac{1}{\mathrm{i}}\left[\frac{1}{w-k_{x}}+\frac{R}{w-k_{x}^{*}}\right]+\frac{\sin 2 \sigma b \hat{f}_{1}(w)}{2 \sigma[\cos 2 \lambda-\cos 2 \sigma b]} \\
& +\frac{\cos \sigma b[1-\exp (-2 \mathrm{i} \lambda)] \hat{f}_{2}(w)}{2[\cos 2 \lambda-\cos 2 \sigma b]} \\
\hat{F}_{1}(w)= & {\left[\frac{k_{y} \exp \left[\mathrm{i}\left(a k_{x}+b k_{y}\right)\right.}{w-k_{x}}+\frac{R k_{y}^{*} \exp \left[\mathrm { i } \left(a k_{x}^{*}+b k_{y}^{*}\right.\right.}{w-k_{x}^{*}}\right] \exp (-\mathrm{i} a w) } \\
& -\frac{\cos \sigma b[1-\exp (2 \mathrm{i} \lambda)] \hat{f}_{1}(w)}{2[\cos 2 \lambda-\cos 2 \sigma b]}-\frac{\sigma \sin 2 \sigma b \hat{f}_{2}(w)}{2[\cos 2 \lambda-\cos 2 \sigma b]} .
\end{aligned}
$$

Now we note that both $\hat{F}_{1}(w)$ and $\hat{f}_{2}(w)$ possess exponential behaviour. That is

$$
\begin{aligned}
\hat{f}_{2}(w) & =\int_{a}^{\infty} f_{2}(x) \exp (-\mathrm{i} w x) \mathrm{d} x=\int_{0}^{\infty} \exp [-\mathrm{i} w(t+a)] f_{2}(t+a) \mathrm{d} t \\
& =\exp (-\mathrm{i} a w) \int_{0}^{\infty} \exp (-i t w) f_{2}(t+a) \mathrm{d} t=\hat{f}_{2}^{*}(w) \exp (-\mathrm{i} a w) .
\end{aligned}
$$

Similarly, $\hat{F}_{1}(w)=\exp (-i a w) \hat{F}_{1}^{*}(w)$, where

$$
\hat{F}_{1}^{*}(w)=\int_{-\infty}^{0} \exp [-\mathrm{i} t w] \hat{F}_{1}(t+a) \mathrm{d} t .
$$

Hence in terms of $\hat{F}_{1}^{*}(w)$ and $\hat{f}_{2}^{*}(w)$ we have

$$
\begin{aligned}
\hat{F}_{2}(w)= & \frac{1}{\mathrm{i}}\left[\frac{1}{w-k_{x}}+\frac{R}{w-k_{x}^{*}}\right]+\frac{\sin 2 \sigma b \hat{f}_{1}(w)}{2 \sigma[\cos 2 \lambda-\cos 2 \sigma b]} \\
& +\frac{\mathrm{i} \cos \sigma b \sin \lambda \exp (-\mathrm{i} k \rho c) \hat{f}_{2}^{*}(w)}{[\cos 2 \hat{\lambda}-\cos 2 \sigma b]}
\end{aligned}
$$


and

$$
\begin{aligned}
\hat{F}_{1}^{*}(w) \exp (-i k \rho c)= & {\left[\frac{k_{y}}{w-k_{x}}+\frac{R k_{y}^{*}}{w-k_{x}^{*}}\right]+\frac{i \sin \lambda \cos \sigma b \hat{f}_{1}(w)}{[\cos 2 \lambda-\cos 2 \sigma b]} } \\
& -\frac{\sigma \sin 2 \sigma b \hat{f}_{2}^{*}(w) \exp (-i k \rho c)}{2[\cos 2 \lambda-\cos 2 \sigma b]} .
\end{aligned}
$$

In matrix notation we have

$$
\begin{aligned}
& \frac{\cos \sigma b}{[\cos 2 \lambda-\cos 2 \sigma b]}\left[\begin{array}{cc}
\mathrm{i} \sin \lambda & -\sigma \sin \sigma b \\
\sin \sigma b & \mathrm{i} \sin \lambda
\end{array}\right]\left[\begin{array}{c}
\hat{f}_{1}(w) \\
\exp (-\mathrm{i} k \rho c) \hat{f}_{2}^{*}(w)
\end{array}\right] \\
& =\left[\begin{array}{c}
-k_{y} \\
\mathrm{i}
\end{array}\right] \frac{1}{\left(w-k_{x}\right)}+R\left[\begin{array}{c}
-k_{y}^{*} \\
\mathrm{i}
\end{array}\right] \frac{1}{\left(w-k_{x}^{*}\right)}+\left[\begin{array}{c}
\hat{F}_{1}^{*}(w) \exp (-\mathrm{i} k \rho c) \\
\hat{F}_{2}(w)
\end{array}\right],
\end{aligned}
$$

where $\rho=\cos (\theta-\alpha)$ since $k_{x}^{*}=k \cos (2 \alpha-\theta)$ and $k_{y}^{*}=k \sin (2 \alpha-\theta)$. We write henceforth

$$
\mathbf{C}(w)=\left[\begin{array}{cc}
\frac{\sin \lambda}{\sqrt{\left(\sin ^{2} \lambda-\sin ^{2} \sigma b\right)}} & \frac{\mathrm{i} \sigma \sin \sigma b}{\sqrt{\left(\sin ^{2} \lambda-\sin ^{2} \sigma b\right)}} \\
-\frac{\sin \sigma b}{\sigma} \frac{\sin \lambda}{\sqrt{\left(\sin ^{2} \lambda-\sin ^{2} \sigma b\right)}} & \frac{\left.\sin \sin ^{2} \lambda-\sin ^{2} \sigma b\right)}{\sqrt{\left(\sin ^{2}\right.}}
\end{array}\right]
$$

and

$$
K(w)=\frac{\cos \sigma b}{\sqrt{\left(\sin ^{2} \lambda-\sin ^{2} \sigma b\right)}}=\frac{L(w)}{M(w)}
$$

where $L(w)=\cos \sigma b$ and $M(w)=\sqrt{ }\left(\sin ^{2} \lambda-\sin ^{2} \sigma b\right)$. The matrix $\mathbf{C}(w)$ has been written in the above form to make the determinant of $\mathbf{C}(w)$ equal to one. With this notation (3.3) becomes

where

$$
K(w) \mathbf{C}(w) \hat{\mathbf{f}}(w) / 2=\phi^{*}(w)+\hat{\mathbf{F}}(w)
$$

$$
\hat{\mathbf{f}}(w)=\left[\begin{array}{c}
\hat{f}_{1}(w) \\
\exp (-\mathrm{i} k \rho c) \hat{f}_{2}^{*}(w)
\end{array}\right], \quad \hat{\mathbf{F}}(w)=\mathrm{i}\left[\begin{array}{c}
\hat{F}_{1}^{*}(w) \exp (-\mathrm{i} k \rho c) \\
\hat{F}_{2}(w)
\end{array}\right]
$$

and

$$
\phi^{*}(\mathbf{w})=-\left[\begin{array}{c}
i k_{y} \\
1
\end{array}\right] \frac{1}{\left(w-k_{x}\right)}-R\left[\begin{array}{c}
i k_{y}^{*} \\
1
\end{array}\right] \frac{1}{\left(w-k_{x}^{*}\right)} .
$$

The equations in (3.4) are analytic in the strip $-\varepsilon<\operatorname{Im} w<0$ where the constant $\varepsilon$ has been defined in Section 2 . That is, all the terms in this system are analytic in this strip.

\section{The factoring of the matrix $C(w)$ and the scalar functions $L(w)$ and $M(w)$}

Let us suppose that we can factor the matrix $\mathbf{C}(w)$ into two matrices $\mathbf{C}_{+}(w)$ and $\mathbf{C}_{-}(w)$ where $\mathbf{C}_{+}(w)$ and its inverse are analytic in the upper half-plane $\operatorname{Im} w>-\varepsilon$ and $\varepsilon$ is the smaller of the two quantities $b\left[(\pi-k c \rho)^{2}-c^{2} k^{2}\right]^{1 / 2} / c^{2}>0$ or $b\left[(\pi+k c \rho)^{2}\right.$ $\left.-c^{2} k^{2}\right]^{1 / 2} / c^{2}>0$ while $C_{-}(w)$ and its inverse are analytic in the lower half-plane 
Im $w<0$. If this is the case and $\mathbf{C}_{+}(w) \mathbf{C}_{-}(w)=\mathbf{C}(w)$ we will be in a position to apply the original argument of Wiener and Hopf to the system of equations (3.3). We will show that this decomposition of $\mathbf{C}(w)$ can be accomplished with Sylvester's theorem. ${ }^{5}$

To start with we write the matrix $\mathbf{C}(w)=\exp [\mathbf{B}(w)]$ and decompose $\mathbf{B}(w)$ additively into $\mathbf{B}_{+}(w)+\mathbf{B}_{-}(w)$, where $\mathbf{B}_{+}(w)$ possess the same half-planes of analyticity which are required of $\mathbf{C}_{+}(w)$ and $\mathbf{C}_{-}(w)$ and furthermore commute. We have from Sylvester's theorem

$$
\begin{aligned}
\mathbf{B}(w)= & \ln \mathbf{C}(w)=\frac{1}{2} \ln \frac{(\sin \lambda+\sin \sigma b)}{\sqrt{\left(\sin ^{2} \lambda-\sin ^{2} \sigma b\right)}}\left[\begin{array}{cc}
1 & \mathrm{i} \sigma \\
-\frac{\mathrm{i}}{\sigma} & 1
\end{array}\right] \\
& +\frac{1}{2} \ln \frac{(\sin \lambda-\sin \sigma b)}{\sqrt{\left(\sin ^{2} \lambda-\sin ^{2} \sigma b\right)}}\left[\begin{array}{cc}
1 & -\mathrm{i} \sigma \\
\frac{\mathrm{i}}{\sigma} & 1
\end{array}\right] \\
= & \ln \left\{\frac{\sin \lambda+\sin \sigma b}{\sin \lambda-\sin \sigma b}\right\}^{1 / 2}\left[\begin{array}{cc}
0 & \mathrm{i} \sigma \\
-\frac{\mathrm{i}}{\sigma} & 0
\end{array}\right]
\end{aligned}
$$

Hence

$$
\mathbf{C}(w)=\exp \left\{\ln \left[\frac{\sin \lambda+\sin \sigma b}{\sin \lambda-\sin \sigma b}\right]^{1 / 2}\left[\begin{array}{cc}
0 & \mathrm{i} \sigma \\
-\frac{\mathrm{i}}{\sigma} & 0
\end{array}\right]\right\}
$$

It therefore remains to decompose the elements of the matrix

$$
\ln \left[\frac{\sin \lambda+\sin \sigma b}{\sin \lambda-\sin \sigma b}\right]^{1 / 2}\left[\begin{array}{cc}
0 & \mathrm{i} \sigma \\
-\frac{\mathrm{i}}{\sigma} & 0
\end{array}\right]
$$

additively. As we shall see in the Appendix this may be rewritten as

$$
\left[g_{+}(w)+g_{-}(w)\right]\left[\begin{array}{cc}
0 & \mathrm{i} \sigma \\
-\frac{\mathrm{i}}{\sigma} & 0
\end{array}\right]
$$

where $\sigma g_{+}(w)$ and $g_{+}(w) / \sigma$ have the same half-plane of analyticity which $\mathbf{C}_{+}(w)$ possesses, whereas $\sigma g_{-}(w)$ and $g_{-}(w) / \sigma$ have the same half-plane of analyticity which $\mathbf{C}_{-}(w)$ possesses. This last reduction is what makes it possible to produce explicit results, in part due to the matrix factors and in part due to the relatively simple form of $g_{+}(w)$ and $g_{-}(w)$. We note that

$$
\left[\begin{array}{cc}
0 & \mathrm{i} \sigma \\
-\frac{\mathrm{i}}{\sigma} & 0
\end{array}\right]^{2}=\mathbf{I}
$$


We shall derive $g_{-}(w), g_{+}(w)$ in the Appendix and, for the time being, we merely present $g_{+}(w)$ for which we have immediate use. We have that

$$
\begin{aligned}
g_{+}(w)= & \frac{1}{2} \ln \prod_{n=1}^{\infty}\left\{\frac{k^{2}-w w_{2 n}^{+}+\sigma(w) \sigma\left(w_{2 n}^{+}\right)}{k^{2}-w w_{2 n}^{-}+\sigma(w)\left(w_{2 n}^{-}\right)} \frac{w-w_{2 n}^{-}}{w-w_{2 n}^{+}}\right\}^{2} \\
& \times\left\{\frac{k^{2}-w w_{n}^{-}+\sigma(w) \sigma\left(w_{n}^{-}\right)}{k^{2}-w w_{n}^{+}+\sigma(w) \sigma\left(w_{n}^{+}\right)} \frac{w-w_{n}^{+}}{w-w_{n}^{-}}\right\},
\end{aligned}
$$

where

$$
w_{n}^{--}=-\frac{a(n \pi-k \rho c)+\mathrm{i} b \sqrt{\left[(n \pi-k \rho c)^{2}-c^{2} k^{2}\right]}}{c^{2}}
$$

and

$$
w_{n}^{+}=\frac{a(n \pi+k \rho c)-\mathrm{i} b \sqrt{\left[(n \pi+k \rho c)^{2}-c^{2} k^{2}\right]}}{c^{2}}, \quad n=1,2, \ldots
$$

The need for the restriction that $\Delta( \pm \rho, 1)=\left[(\pi \pm \rho c)^{2}-c^{2} k^{2}\right]^{1 / 2}$ is real, is now clear. This condition precludes the presence of diffracted plane waves from the structure in Figure 1. We note at this point, and shall show in the Appendix that $g_{+}(w)$ is single valued in the smaller of the half-planes $\operatorname{Im} w>-b\left[(\pi+k \rho c)^{2}-c^{2} k^{2}\right]^{1 / 2} / c^{2}$ or $-b\left[(\pi-k \rho c)^{2}-c^{2} k^{2}\right]^{1 / 2} / c^{2}$.

The scalar factor $[\cos 2 \sigma b-\cos 2 \lambda]^{1 / 2} / \sqrt{ } 2$ was encountered in Reference 1 , and it is known how to decompose it into a product representation $M_{-}(w) / M_{+}(w)$. This expression has been chosen to have a positive square root in the interval $k_{x}^{*}<u<$ $k_{x}(w=u+i v)$. The factor $M_{-}(w)$ is free of branch points in the lower half-plane $\operatorname{Im} w<0$ and the factor $1 / M_{+}(w)$ is free of branch points in the upper half-plane $\operatorname{Im} w>-\varepsilon$.

The factor $L(w)=\cos \sigma b$ has no zeros or poles in the strip $-\varepsilon<\operatorname{Im} w<0$, if the parameter $k b$ is properly restricted. The zeros of $\cos \sigma b$ are related to the propagation constants in the ducts, and since we only want one mode to propagate, we have $\pi / 2 b<k<3 \pi / 2 b$. On the other hand, since there are no diffracted plane waves, $[(\pi$ $\left.\pm k \rho c)^{2}-c^{2} k^{2}\right]$ is positive. Upon taking into account all of these conditions, we see that a diffracted plane wave can occur before the propagation interval in the duct is completed. Hence the factors of $L(w)$ which we require are $L_{-}(w)$ and $L_{+}(w)$, where $L_{-}(w)$ is free of zeros in the lower half-plane $\operatorname{Im} w<0$ and $1 / L_{+}(w)$ is free of poles in the upper half-plane

$$
\operatorname{lm} w>-\left[\frac{9 \pi^{2}}{4 b^{2}}-k^{2}\right]^{1 / 2} .
$$

\section{The solution of the system (3.4)}

We can now write the system (3.4) as

$$
\frac{1}{2} \frac{K_{-}(w)}{K_{+}(w)} \mathbf{C}_{+}(w) \mathbf{C}_{-}(w) \hat{\mathbf{f}}(w)=\phi^{*}(w)+\hat{\mathbf{F}}(w),
$$

which is analytic in the strip $-\varepsilon<\operatorname{Im} w<0$. This in turn can be rewritten as

$$
\frac{1}{2} K_{-}(w) \mathbf{C}_{-}(w) \hat{\mathbf{f}}(w)=K_{+}(w) \mathbf{C}_{+}^{-1}(w) \phi^{*}(w)+K_{+}(w) \mathbf{C}_{+}^{-1}(w) \hat{\mathbf{F}}(w),
$$


where now the left side of this matrix equation is analytic in the lower half-plane $\dagger$ $\operatorname{Im} w<0$ and the extreme right term is analytic in the upper half-plane $\operatorname{Im} w>-\varepsilon$. Owing to the simplicity of $\phi^{*}(w)$, it is possible to decompose the middle term explicitly into two terms with half-planes of analyticity $\operatorname{Im} w<0$ and $\operatorname{Im} w>-\varepsilon$. Hence we obtain

$$
\begin{aligned}
& K_{-}(w) \mathbf{C}_{-}(w) \hat{\mathbf{f}}(w) / 2+\frac{K_{+}\left(k_{x}\right) \mathbf{C}_{+}^{-1}\left(k_{x}\right)}{\left(w-k_{x}\right)}\left[\begin{array}{c}
\mathrm{i} k_{y} \\
1
\end{array}\right]+\frac{R K_{+}\left(k_{x}^{*}\right) \mathbf{C}_{+}^{-1}\left(k_{x}^{*}\right)}{\left(w-k_{x}^{*}\right)}\left[\begin{array}{c}
\mathrm{i} k_{y}^{*} \\
1
\end{array}\right] \\
& =-\frac{\left[K_{+}(w) \mathbf{C}_{+}^{-1}(w)-K_{+}\left(k_{x}\right) \mathbf{C}_{+}^{-1}\left(k_{x}\right)\right]}{\left(w-k_{x}\right)}\left[\begin{array}{c}
\mathrm{i} k_{y} \\
1
\end{array}\right] \\
& -R \frac{\left[K_{+}(w) \mathbf{C}_{+}^{-1}(w)-K_{+}\left(k_{x}^{*}\right) C_{+}^{-1}\left(k_{x}^{*}\right)\right]}{\left(w-k_{x}^{*}\right)}\left[\begin{array}{c}
\mathrm{i} k_{y}^{*} \\
1
\end{array}\right]+K_{+}(w) \mathbf{C}_{+}^{-1}(w) \hat{\mathbf{F}}(w) .
\end{aligned}
$$

Now the original argument of Wiener and Hopf may be invoked. That is, the left and right sides are analytic in a common strip, with the left side analytic in the lower half plane $\operatorname{Im} w<0$ and the right sides analytic in the upper half-plane $\operatorname{Im} w>-\varepsilon$. The right side is therefore the analytic continuation of the left side and is analytic everywhere. We have therefore

$$
\begin{aligned}
K_{-}(w) \mathbf{C}_{-}(w) \hat{\mathbf{f}}(w) / 2 & +\frac{K_{+}\left(k_{x}\right) \mathbf{C}_{+}^{-1}\left(k_{x}\right)}{\left(w-k_{x}\right)}\left[\begin{array}{c}
\mathrm{i} k_{y} \\
1
\end{array}\right] \\
& +\frac{R K_{+}\left(k_{x}^{*}\right) \mathbf{C}_{+}^{-1}\left(k_{x}^{*}\right)}{\left(w-k_{x}^{*}\right)}\left[\begin{array}{c}
\mathrm{i} k_{y}^{*} \\
1
\end{array}\right]=\left[\begin{array}{c}
E_{1}(w) \\
E_{2}(w)
\end{array}\right]
\end{aligned}
$$

and

$$
\begin{aligned}
K_{+}(w) \mathbf{C}_{+}^{-1}(w) \hat{\mathbf{F}}(w)-\frac{\left[K_{+}(w) \mathbf{C}_{+}^{-1}(w)-K_{+}\left(k_{x}\right) \mathbf{C}_{+}^{-1}\left(k_{x}\right)\right]}{\left(w-k_{x}\right)}\left[\begin{array}{c}
\mathrm{i} k_{y} \\
1
\end{array}\right] \\
-R \frac{\left[K_{+}(w) \mathbf{C}_{+}^{-1}(w)-K_{+}\left(k_{x}^{*}\right) \mathbf{C}_{+}^{-1}\left(k_{x}^{*}\right)\right]}{\left(w-k_{x}^{*}\right)}\left[\begin{array}{c}
\mathrm{i} k_{y}^{*} \\
1
\end{array}\right]=\left[\begin{array}{c}
E_{1}(w) \\
E_{2}(w)
\end{array}\right],
\end{aligned}
$$

where $E_{1}(w)$ and $E_{2}(w)$ are entire functions of $w$.

In order to determine these entire functions, we note that $f_{1}(x)=O\left(x^{-1 / 2}\right)$ and $f_{2}^{*}(x)=O(1), x \rightarrow 0+$. Upon taking into account the properties of $f_{1}(x)$ and $f_{2}(x)$ for $0<x<\infty$, we find that $\hat{f}_{1}(w)=O\left(w^{-1 / 2}\right)$ and $f_{2}^{*}(w)=O\left(w^{-1}\right),|w| \rightarrow \infty, \operatorname{Im} w<0$. Furthermore (see Appendix B)

$$
\mathbf{C}_{-}(w)=\left[\begin{array}{cc}
O(1) & O(w) \\
O(1 / w) & O(1)
\end{array}\right],
$$

$|w| \rightarrow \infty, \operatorname{Im} w<0$, and $K_{-}(w)=O\left(w^{1 / 2}\right),|w| \rightarrow \infty, \operatorname{Im} w<0$. Hence from (5.3) we see that $E_{1}(w)$ is bounded at infinity in the lower half-plane $\operatorname{Im} w<0$, whereas $E_{2}(w)$ vanishes there. Upon examining equation (5.4) we find that $E_{1}(w)$ is bounded at

\footnotetext{
†When we say that such a matrix is analytic in some half-plane, we mean that its elements, its determinant and the reciprocal of the determinant are analytic there.
} 
infinity in the upper half-plane $\operatorname{Im} w>-\varepsilon$ whereas $E_{2}(w)$ vanishes there. Hence by Liouville's theorem $E_{1}(w)$ is a constant $\delta$ and $E_{2} \equiv 0$.

It remains to evaluate the constant $\delta$. We recall that the structure in Fig. 1 has been excited by a plane wave. The ducts therefore sustain waves propagating to the right. The solution for the vector $\hat{f}(w)$ contains terms which account for waves propagating to the right and left and are due to the real zeros in $K_{-}(w)$ at $w= \pm \kappa=\left\{k^{2}\right.$ $\left.-(\pi / 2 b)^{2}\right\}^{1 / 2}$. In order to eliminate the possibility of waves from the right in the duct, we require that

$$
\frac{K_{+}\left(k_{x}\right) \mathbf{C}_{+}^{-1}\left(k_{x}\right)}{\left(\kappa+k_{x}\right)}\left[\begin{array}{c}
\mathrm{i} k_{y} \\
1
\end{array}\right]+\frac{R K_{+}\left(k_{x}^{*}\right) \mathbf{C}_{+}^{-1}\left(k_{x}^{*}\right)}{\left(\kappa+k_{x}^{*}\right)}\left[\begin{array}{c}
\mathrm{i} k_{y}^{*} \\
1
\end{array}\right]+\left[\begin{array}{l}
\delta \\
0
\end{array}\right]=\mathbf{0}
$$

That is, the residue of the vector $\hat{\mathbf{f}}(w)$ at $w=-\kappa$ vanishes. Equation (5.5) enables us to evaluate the reflection coefficient $R$ and the constant $\delta$. We have

and

$$
R=-\frac{\left(\kappa+k_{x}^{*}\right) K_{+}\left(k_{x}\right) \exp \left[g_{+}\left(k_{x}^{*}\right)\right]}{\left(\kappa+k_{x}\right) K_{+}\left(k_{x}^{*}\right) \exp \left[g_{+}\left(k_{x}\right)\right]}
$$

$$
\delta=-\frac{\mathrm{i}\left(k_{y}-k_{y}^{*}\right) K_{+}\left(k_{x}\right) \exp \left[-g_{+}\left(k_{x}\right)\right]}{\left(\kappa+k_{x}\right)} .
$$

We shall have more to say about the far field parameters in the next section.

\section{The determination of the reflection and transmission coefficients}

The determination of the reflection coefficient is immediate. We obtain in the Appendix explicit forms of the various terms in the formula (5.6) for $R$. Here we shall give its magnitude and although it is possible to write its argument, we will not do it since we have no direct use for it. We have then

$$
|R|=\left[\frac{\left(\kappa-k_{x}\right)\left(\kappa+k_{x}^{*}\right)}{\left(\kappa+k_{x}\right)\left(\kappa-k_{x}^{*}\right)}\right]^{1 / 2} \text {. }
$$

The determination of the transmission coefficients in the ducts requires much more effort. We note that because of the periodic nature of the structure, we need only examine the region $-\infty<x<\infty, 0 \leq y \leq 2 b$. The transmission coefficient in the duct $x>a, 0 \leq y \leq b$ is different from one in the duct $x>2 a, b \leq y \leq 2 b$ for we have two different forms for the representation (2.3) based upon (2.4). For example, for $0 \leq y \leq b$, we have from (2.3) and (2.4) the Fourier integral representation

$$
\begin{aligned}
\phi(x, y)= & \exp \left[\mathrm{i}\left(x k_{x}+y k_{y}\right)\right]+R \exp \left[\mathrm{i}\left(x k_{x}^{*}+y k_{y}^{*}\right]\right. \\
& +\frac{1}{2 \pi} \int_{\mathscr{L}} \frac{\{\exp [2 \mathrm{i} \lambda(w) \sin \sigma y+\sin \sigma(2 b-y)\}}{2 \sigma[\cos 2 \lambda(w)-\cos 2 \sigma b]} \widehat{f}_{1}(w) \exp (\mathrm{i} w x) \mathrm{d} w \\
& +\frac{1}{2 \pi} \int_{\mathscr{T}} \frac{\{\cos \sigma(b+y)-\exp [-2 \mathrm{i} \lambda(w)] \cos \sigma(b-y)\}}{2[\cos 2 \lambda(w)-\cos 2 \sigma b]} \\
& \times \hat{f}_{2}(w) \exp (\mathrm{i} w x) \mathrm{d} w,
\end{aligned}
$$

where $\mathscr{L}$ is a line drawn in the strip $-\varepsilon<\operatorname{Im} w<0$, parallel to the real axis of the $w$ plane and where $\varepsilon$ has been defined in Section 2. For $b \leq y \leq 2 b$, we have 


$$
\begin{aligned}
\phi(x, y)= & \exp \left[\mathrm{i}\left(x k_{x}+y k_{y}\right)\right]+R \exp \left[\mathrm{i}\left(x k_{x}^{*}+y k_{y}^{*}\right)\right. \\
& +\frac{1}{2 \pi} \int_{\mathscr{L}} \frac{\{\exp [2 \mathrm{i} \lambda(w)] \sin \sigma y+\sin \sigma(2 b-y)\}}{2 \sigma[\cos 2 \lambda(w)-\cos 2 \sigma b]} \hat{f}_{1}(w) \exp (\mathrm{i} w x) \mathrm{d} w \\
& +\frac{1}{2 \pi} \int_{\mathscr{L}} \frac{\{\exp [2 \mathrm{i} \lambda(w)] \cos \sigma(b-y)-\cos \sigma(3 b-y)\}}{2[\cos 2 \hat{\lambda}(w)-\cos 2 \sigma b]} \\
& \times \hat{f}_{2}(w) \exp (\mathrm{i} w x) \mathrm{d} w .
\end{aligned}
$$

In order to examine (6.1) and (6.2) in more detail, we need the explicit forms of $\mathbf{C}_{+}(w)$ and $\mathbf{C}_{-}(w)$, so that $\hat{f}_{1}(w)$ and $\hat{f}_{2}(w)$ may be determined. Here

$$
\mathbf{C}_{+}(w)=\left[\begin{array}{cc}
\cosh g_{+}(w) & \mathrm{i} \sigma \sinh g_{+}(w) \\
-\frac{\mathrm{i}}{\sigma} \sinh g_{+}(w) & \cosh g_{+}(w)
\end{array}\right]
$$

and therefore

$$
\mathbf{C}_{+}^{-1}(w)=\left[\begin{array}{cc}
\cosh g_{+}(w) & -\mathrm{i} \sigma \sinh g_{+}(w) \\
\frac{\mathrm{i}}{\sigma} \sinh g_{+}(w) & \cosh g_{+}(w)
\end{array}\right],
$$

where $g_{+}(w)$ is given in the Appendix. It follows that

and

$$
\mathbf{C}_{+}^{-1}\left(k_{x}\right)\left[\begin{array}{c}
\mathrm{i} k_{y} \\
1
\end{array}\right]=\left[\begin{array}{c}
\mathrm{i} k_{y} \\
1
\end{array}\right] \exp \left[-g_{+}\left(k_{x}\right)\right]
$$

$$
\mathbf{C}_{+}^{-1}\left(k_{x}^{*}\right)\left[\begin{array}{c}
i k_{y}^{*} \\
1
\end{array}\right]=\left[\begin{array}{c}
i k_{y}^{*} \\
1
\end{array}\right] \exp \left[-g_{+}\left(k_{x}^{*}\right)\right] \text {. }
$$

Hence

$$
\begin{aligned}
\frac{K_{-}(w) \mathbf{C}_{-}(w) \hat{\mathbf{f}}(w)}{2}= & -\frac{K_{+}\left(k_{x}\right) \exp \left[-g_{+}\left(k_{x}\right)\right]}{w-k_{x}}\left[\begin{array}{c}
\mathrm{i} k_{y} \\
1
\end{array}\right] \\
& -\frac{R K_{+}\left(k_{x}^{*}\right) \exp \left[-g_{+}\left(k_{x}^{*}\right)\right]}{w-k_{x}^{*}}\left[\begin{array}{c}
\mathrm{i} k_{y}^{*} \\
1
\end{array}\right]+\left[\begin{array}{c}
\delta \\
0
\end{array}\right] .
\end{aligned}
$$

With our knowledge of $R$ and $\delta$, the right side of (6.3) can be rewritten as

$$
-\frac{K_{+}\left(k_{x}\right)(w+\kappa) \exp \left[-g_{+}\left(k_{x}\right)\right]}{\left(w-k_{x}\right)\left(w-k_{x}^{*}\right)\left(\kappa+k_{x}\right)}\left[\begin{array}{c}
\mathrm{i}\left[w\left(k_{y}-k_{y}^{*}\right)+\left(k_{x} k_{y}^{*}-k_{y} k_{x}^{*}\right)\right] \\
\left(k_{x}-k_{x}^{*}\right)
\end{array}\right],
$$

but the column matrix may be simplified to

$$
\frac{2 k \sin (\alpha-\theta)}{c}\left[\begin{array}{c}
i \lambda(w) \\
b
\end{array}\right]
$$

and therefore

$$
\hat{\mathbf{f}}(w)=-\frac{4 K_{+}\left(k_{x}\right)(w+\kappa) k \sin (\alpha-\theta) \exp \left[-g_{+}\left(k_{x}\right)\right]}{c K_{-}(w)\left(w-k_{x}\right)\left(w-k_{x}^{*}\right)\left(\kappa+k_{x}\right)} \mathbf{C}_{-}^{-1}(w)\left[\begin{array}{c}
\mathrm{i} \lambda(w) \\
b
\end{array}\right]
$$


This incidently shows that $f_{1}(x)$ and $f_{2}(x)$ are integrable in the neighbourhood of the origin. Upon using the fact that

$$
g_{-}(w)+g_{+}(w)=\ln \left[\frac{\sin \lambda(w)+\sin \sigma b}{\sin \lambda(w)-\sin \sigma b}\right]^{1 / 2}
$$

we may rewrite (6.4) in component form as

and

$$
\begin{aligned}
\hat{f}_{1}(w)= & \frac{\mathrm{i} A(w)\left[\sin ^{2} \lambda(w)-\sin ^{2} \sigma b\right]^{1 / 2}}{\cos \sigma b}\left\{\left[\frac{\sin \lambda(w)+\sin \sigma b}{\sin \lambda(w)-\sin \sigma b}\right]^{1 / 2}\right. \\
& \times[\lambda(w)-\sigma b] \exp \left[-g_{+}(w)\right]+\left[\frac{\sin \lambda(w)-\sin \sigma b}{\sin \lambda(w)+\sin \sigma b}\right]^{1 / 2} \\
& \left.\times[\lambda(w)+\sigma b] \exp \left[g_{+}(w)\right]\right\}
\end{aligned}
$$

$$
\begin{aligned}
\hat{f}_{2}^{*}(w)= & \frac{\exp (i k \rho c) A(w)\left[\sin ^{2} \lambda(w)-\sin ^{2} \sigma b\right]^{1 / 2}}{\cos \sigma b}\left\{-\left[\frac{\sin \lambda(w)+\sin \sigma b}{\sin \lambda(w)-\sin \sigma b}\right]^{1 / 2}\right. \\
& \times\left[\frac{\lambda(w)-\sigma b}{\sigma}\right] \exp \left[-g_{+}(w)\right]+\left[\frac{\sin \lambda(w)-\sin \sigma b}{\sin \lambda(w)+\sin \sigma b}\right]^{1 / 2} \\
& \times\left[\frac{\lambda(w)+\sigma b}{\sigma}\right] \exp \left[g_{+}(w)\right\} .
\end{aligned}
$$

These forms exhibit explicitly the dependence of $\hat{f}_{1}(w)$ and $\hat{f}_{2}^{*}(w)$ on the poles $w= \pm \kappa$. Here

and

$$
A(w)=\frac{2 k(w+\kappa) \sin (\alpha-\theta) K_{+}\left(k_{x}\right) \exp \left[-g_{+}\left(k_{x}\right)\right]}{c K_{+}(w)\left(w-k_{x}\right)\left(w-k_{x}^{*}\right)\left(\kappa+k_{x}\right)}
$$

$$
\frac{K_{-}(w)}{K_{+}(w)}=\frac{\cos \sigma b}{\left[\sin ^{2} \lambda(w)-\sin ^{2} \sigma b\right]^{1 / 2}} .
$$

Upon substituting $\hat{f}_{1}(w)$ and $\hat{f}_{2}(w)=\exp (-\mathrm{i} w a) \hat{f}_{2}^{*}(w)$ into $(6.1)$ we observe that the integrand is a single-valued function of $w$, a fact which we shall discuss in the Appendix. For $x<0,0 \leq y \leq 2 b$ we may close $\mathscr{L}$ in the lower half-plane by a sequence of semicircular arcs which pass between the double set of poles of the integrand. Since there are no real poles in this half-plane, it follows that $\phi(x, y)$ is asymptotic to

$$
\exp \left[\mathrm{i}\left(x k_{x}+y k_{y}\right)\right]+R \exp \left[\mathrm{i}\left(x k_{x}^{*}+y k_{y}^{*}\right)\right] .
$$

If we close $\mathscr{L}$ in the upper half-plane by a sequence of semicircular arcs which pass between the double set of poles of the integrand, we obtain residues from the complex poles as well as from three real ones, namely $k_{x}, k_{x}^{*}$ and $\kappa$. The residues from the complex poles give rise to terms which vanish exponentially when $x \rightarrow \infty$. The residues from the poles at $k_{x}$ and $k_{x}^{*}$ give rise to terms which cancel the term (6.5), whereas the residue from the pole at $\kappa$ enables us to determine the amplitudes (not the transmission coefficients) of the propagating waves in the ducts $x>2 a, 0 \leq y \leq 2 b$. A residue 
calculation gives us for $x \rightarrow \infty, 0 \leq y \leq b$

$$
\begin{aligned}
\phi(x, y)= & \frac{+\mathrm{i}\left\{b\left[1+\exp (2 \mathrm{i} \lambda(\kappa)) \operatorname{Res}_{w=\kappa} \hat{f}_{1}(w) \sin \frac{\pi y}{2 b} \exp (\mathrm{i} \kappa x)\right\}\right.}{\pi[1+\cos 2 \lambda(\kappa)]} \\
& \times \frac{-\mathrm{i}\left\{[1+\exp (-2 \mathrm{i} \lambda(\kappa))] \underset{w=\kappa}{\operatorname{Res}} \hat{f}_{2}(w) \sin \frac{\pi y}{2 b} \exp (\mathrm{i} \kappa x)\right\}}{2[1+\cos 2 \lambda(\kappa)]} \\
\phi(x, y)= & \frac{A(\kappa)\left[\left(\pi^{2} / 4\right)-\lambda^{2}(\kappa)\right]^{1 / 4}}{\kappa b[\cos \lambda(\kappa)]^{1 / 2}}\left\{[1+\mathrm{i} \exp \mathrm{i} \lambda(\kappa)][1+\sin \lambda(\kappa)]^{1 / 2}\right. \\
& \times[(\pi / 2)-\lambda(\kappa)]^{1 / 2} \exp [-\mathrm{i} v(\kappa)]+[\mathrm{i} \exp \mathrm{i} \lambda(\kappa)-1][1-\sin \lambda(\kappa)]^{1 / 2} \\
& \left.\times[(\pi / 2)+\lambda(\kappa)]^{1 / 2} \exp [\mathrm{i} v(\kappa)]\right\} \sin \frac{\pi y}{2 b} \exp [\mathrm{i} \kappa(x)] .
\end{aligned}
$$

As we shall see in the Appendix

$$
|A(\kappa)|=\frac{4 c^{1 / 2} k \kappa \sin \alpha \sin (\alpha-\theta)}{\pi^{1 / 2}[\cos \lambda(\kappa)]^{1 / 2}\left[k_{x}-\kappa\right]^{1 / 4}\left[k_{x}+\kappa\right]^{1 / 2}\left[\kappa-k_{x}^{*}\right]^{3 / 4}} .
$$

Hence the magnitude of the amplitude of the propagating mode in the duct $x>a$, $0 \leq y \leq b$ is

$$
\left|T_{1}\right|=k \sin (\alpha-\theta)\left[\frac{8\left\{\pi-2\left[\left(\pi^{2} / 4\right)-\lambda^{2}(\kappa)\right]^{1 / 2} \sin 2 v(\kappa)\right\}}{\pi\left(k_{x}+\kappa\right)\left(\kappa-k_{x}^{*}\right)}\right]^{1 / 2} .
$$

In a similar fashion, using the representation (6.2), we find that the magnitude of the amplitude of the propagating mode in the duct $x>2 a, b \leq y \leq 2 b$ is

$$
\left|T_{2}\right|=k \sin (\alpha-\theta)\left[\frac{8\left\{\pi+2\left[\left(\pi^{2} / 4\right)-\lambda^{2}(\kappa)\right]^{1 / 2} \sin 2 v(\kappa)\right\}}{\pi\left(k_{x}+\kappa\right)\left(\kappa-k_{x}^{*}\right)}\right]^{1 / 2} .
$$

Now we recall ${ }^{3}$ that $|R|,\left|T_{1}\right|$ and $\left|T_{2}\right|$ are related by a conservation condition, namely

$$
2 k c \sin (\alpha-\theta)\left[1-|R|^{2}\right]=b \kappa\left[\left|T_{1}\right|^{2}+\left|T_{2}\right|^{2}\right] / 2,
$$

which is indeed the case here. Hence the transmission coefficients are

$$
\left[\frac{\kappa \sin \alpha}{4 k \sin (\alpha-\theta)}\right]^{1 / 2}\left|T_{1}\right| \text { and }\left[\frac{\kappa \sin \alpha}{4 k \sin (\alpha-\theta)}\right]^{1 / 2}\left|T_{2}\right| \text {, }
$$

respectively. Observe also that $|R|<1$ since $1-|R|^{2}>0$, and therefore the transmission coefficients are less than one. We also note that

$$
\left(\pi^{2} / 4\right)-\lambda^{2}(\kappa)=c^{2}\left[k_{x}-\kappa\right]\left[\kappa-k_{x}^{*}\right],
$$

which is now expressed in terms of physical parameters. The $v(\kappa)$ in (6.6) is the argument of $g_{+}(\kappa)$.

\section{Appendix}

As we have noted in Section 4, we need a usable form of the factors $L_{-}(w), L_{+}(w)$, $M_{-}(w)$ and $M_{+}(w)$ in order to obtain $K_{-}(w)$ and $K_{+}(w)$. By this we mean that not 
only can we display these terms, but we can also find their asymptotic forms for $|w| \rightarrow \infty$ in their respective half-planes of analyticity. In Section 6 we saw that we also had need for $K_{+}\left(k_{x}\right), K_{+}\left(k_{x}^{*}\right)$ and $K_{+}(\kappa)$ in a reduced form in order to calculate the far-field parameters. The same remarks may be addressed to $g_{+}(w)$ and $g_{-}(w)$ and it is to these issues that we devote our attention here.

\section{A. The decomposition of $K(w)$}

To begin with we require the factors of

$$
\frac{K_{-}(w)}{K_{+}(w)}=\frac{\cos \sigma b}{\left[\sin ^{2} \lambda-\sin ^{2} \sigma b\right]^{1 / 2}}
$$

where $K_{-}(w)$ and its reciprocal are analytic in the lower half-plane $\operatorname{Im} w<0$ and $K_{+}(w)$ and its reciprocal are analytic in the upper half-plane $\operatorname{Im} w>-\varepsilon$ where $\varepsilon$ has been defined in Section 4.

The factoring of the numerator is immediate since it has been encountered in many scalar Wiener-Hopf problems in the past. It is well known that we can write the absolutely convergent Weierstrass product for $\cos \sigma b$ as

$$
\begin{aligned}
\cos \sigma b & =\prod_{n=0}^{\infty}\left[1-\frac{4 \sigma^{2} b^{2}}{(2 n+1)^{2} \pi^{2}}\right] \\
& =\prod_{n=0}^{\infty}\left[1-\frac{4 b^{2} k^{2}}{(2 n+1)^{2} \pi^{2}}+\frac{4 b^{2} w^{2}}{(2 n+1)^{2} \pi^{2}}\right] .
\end{aligned}
$$

In view of the fact that only the lowest mode will be permitted to propagate in the ducts, we have that

$$
\kappa=\left[k^{2}-\pi^{2} / 4 b^{2}\right]^{1 / 2}>0, \quad \pi<2 b k<3 \pi
$$

and

$$
1-4 b^{2} k^{2} /(2 n+1)^{2} \pi^{2}>0, \quad n=1,2, \ldots
$$

We then rewrite the second product as

$$
\begin{aligned}
& \frac{4 b^{2}}{\pi^{2}}\left(w^{2}-\kappa^{2}\right) \prod_{n=1}^{\infty}\left\{\sqrt{ }\left[1-\frac{4 b^{2} k^{2}}{(2 n+1)^{2} \pi^{2}}\right]+\frac{2 \mathrm{i} b w}{(2 n+1) \pi}\right\} \exp [-2 \mathrm{i} b w /(2 n+1) \pi] \\
& \times \prod_{n=1}^{\infty}\left\{\sqrt{ }\left[1-\frac{4 b^{2} k^{2}}{(2 n+1)^{2} \pi^{2}}\right]-\frac{2 \mathrm{i} b w}{(2 n+1) \pi}\right\} \exp [2 \mathrm{i} b w /(2 n+1) \pi] .
\end{aligned}
$$

The exponential factors have been inserted into these products to render them convergent. Hence

$$
\left(w^{2}-\kappa^{2}\right) \prod_{n=1}^{\infty}\left\{\sqrt{ }\left[1-\frac{4 b^{2} k^{2}}{(2 n+1)^{2} \pi^{2}}\right]+\frac{2 i b w}{(2 n+1) \pi}\right\} \exp [-2 \mathrm{i} b w /(2 n+1) \pi]
$$

has no zeros in the lower half-plane $\operatorname{Im} w<0$ and its reciprocal is analytic in this halfplane. Similarly the second product is free of zeros in the upper half-plane $\operatorname{Im} w>-\varepsilon$ 
and its reciprocal is analytic there. Hence we write $\cos \sigma b=L_{-}(w) / L_{+}(w)$ where

$$
\begin{aligned}
L_{-}(w)= & \frac{4 b^{2}}{\pi^{2}}\left(w^{2}-\kappa^{2}\right) \prod_{n=1}^{\infty}\left\{\int\left[1-\frac{4 b^{2} k^{2}}{(2 n+1)^{2} \pi^{2}}\right]\right. \\
& \left.+\frac{2 i b w}{(2 n+1) \pi}\right\} \exp [-2 i b w /(2 n+1) \pi]
\end{aligned}
$$

and

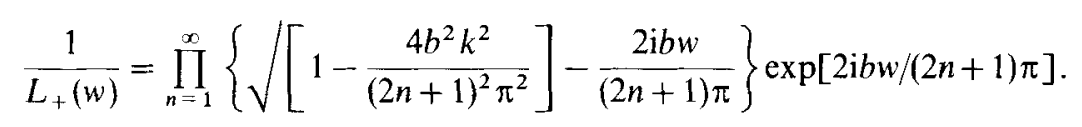

Now as for the asymptotic behaviour of $L_{-}(w)$ and $L_{+}(w)$, we recall ${ }^{1,7}$ that it is equivalent to considering the asymptotic behaviour of

$$
w^{2} \prod_{n=1}^{\infty}[1+2 \mathrm{i} b w /(2 n+1) \pi] \exp [-2 \mathrm{i} b w /(2 n+1) \pi]
$$

in the lower half-plane $\operatorname{Im} w<0,|w| \rightarrow \infty$ and

$$
\prod_{n+1}^{\infty}[1-2 \mathrm{i} b w /(2 n+1) \pi] \exp [2 \mathrm{i} b w /(2 n+1) \pi]
$$

in the upper half-plane Im $w>-\varepsilon,|w| \rightarrow \infty$. These last two products may be expressed in terms of the gamma function. That is, up to a constant

and

$$
L_{-}(w) \sim w \Gamma(\mathrm{i} w b / \pi) \exp [(2-\gamma) \mathrm{i} w b / \pi] / \Gamma(2 \mathrm{i} w b / \pi)
$$

$$
1 / L_{+}(w) \sim \Gamma(-\mathrm{i} w b / \pi) \exp [-(2-\gamma) \mathrm{iw} b / \pi] / w \Gamma(-2 \mathrm{i} w b / \pi),
$$

where $\gamma$ is the Euler-Mascheroni constant.

We now turn to the factors of $\sin ^{2} \lambda-\sin ^{2} \sigma b$. This expression may be rewritten as $\sin (\lambda+\sigma b) \sin (\lambda-\sigma b)$ and has the convergent infinite representation

$$
\left(\lambda^{2}-\sigma^{2} b^{2}\right) \prod_{n=1}^{\infty}\left[1-\left(\frac{\lambda+\sigma b}{n \pi}\right)^{2}\right] \prod_{n=1}^{\infty}\left[1-\left(\frac{\lambda-\sigma b}{n \pi}\right)^{2}\right]=\left[M_{-}(w) / M_{+}(w)\right]^{2}
$$

which appears to be valid in the $w$ plane cut from $-\infty$ to $-k$ and $k$ to $\infty$. But these cuts are illusory since the original expression is single valued and we therefore expect that we can rewrite this last term so that the apparent multivaluedness does not appear. To this end, we may rewrite the last product as

$$
\begin{aligned}
& \left.c^{2}\left(w-k_{x}\right)\left(w-k_{x}^{*}\right)\left\{\prod_{n=1}^{\infty}\left[\left(1-\frac{\lambda}{n \pi}\right)^{2}-\frac{\sigma^{2} b^{2}}{n^{2} \pi^{2}}\right] \exp [2 \lambda / n \pi)\right]\right\} \\
& \left.\times\left\{\prod_{n=1}^{\infty}\left[\left(1+\frac{\lambda}{n \pi}\right)^{2}-\frac{\sigma^{2} b^{2}}{n^{2} \pi^{2}}\right] \exp [-2 \lambda / n \pi)\right]\right\} .
\end{aligned}
$$

Since $\sigma^{2}=k^{2}-w^{2}$ and $\lambda=k c \cos (\theta-\alpha)-a w=k \rho c-a w$, we observe that the infinite products only have complex roots since we have assumed that there is only specular 
reflection of the incident plane wave. Therefore the last display may be rewritten as

$$
\begin{aligned}
{\left[M_{-}(w) / M_{+}(w)\right]^{2}=} & c^{2}\left(w-k_{x}\right)\left(w-k_{x}^{*}\right) \prod_{n=1}^{\infty}\left[\frac{c w}{\pi n}+\frac{a}{c}\left(1-\frac{k \rho c}{\pi n}\right)+\frac{\mathrm{i} b}{c} \Delta(-\rho, n)\right] \\
& \times\left[\frac{c w}{\pi n}+\frac{a}{c}\left(1-\frac{k \rho c}{\pi n}\right)-\frac{\mathrm{i} b}{c} \Delta(-\rho, n)\right] \exp (2 \hat{\lambda} / \pi n) \\
& \times \prod_{n=1}^{\infty}\left[\frac{c w}{\pi n}-\frac{a}{c}\left(1+\frac{k \rho c}{\pi n}\right)+\frac{\mathrm{i} b}{c} \Delta(\rho, n)\right] \\
& \times\left[\frac{c w}{\pi n}-\frac{a}{c}\left(1+\frac{k \rho c}{\pi n}\right)-\frac{\mathrm{i} b}{c} \Delta(\rho, n)\right] \exp (-2 \hat{\lambda} / \pi n),
\end{aligned}
$$

where

$$
\Delta(\rho, n)=\left[\left(1+\frac{k \rho c}{\pi n}\right)^{2}-\frac{c^{2} k^{2}}{\pi^{2} n^{2}}\right]^{1 / 2}>0, \quad n \geq 1 .
$$

In order to have the products in (A1) in a more usable form so that we may determine their asymptotic behaviour, we cast (A1) into the form

$$
\begin{aligned}
& c^{2}\left(w-k_{x}\right)\left(w-k_{x}^{*}\right) \\
& \times \prod_{n=1}^{\infty}\left\{\left[\frac{c w}{\pi n}+\frac{a}{c}\left(1-\frac{k \rho c}{\pi n}\right)+\frac{\mathrm{i} b}{c} \Delta(-\rho, n)\right]\left[\frac{a-\mathrm{i} b}{c}\right]\right. \\
& \left.\times\left[\frac{c w}{\pi n}+\frac{a}{c}\left(1-\frac{k \rho c}{\pi n}\right)-\frac{\mathrm{i} b}{c} \Delta(-\rho, n)\right]\left[\frac{a+\mathrm{i} b}{c}\right]\right\} \exp (2 \lambda / \pi n) \\
& \times\left\{\left[-\frac{c w}{\pi n}+\frac{a}{c}\left(1+\frac{k \rho c}{\pi n}\right)-\frac{\mathrm{i} b}{c} \Delta(\rho, n)\right]\left[\frac{a+\mathrm{i} b}{c}\right]\right. \\
& \left.\times\left[-\frac{c w}{\pi n}+\frac{a}{c}\left(1+\frac{k \rho c}{\pi n}\right)+\frac{\mathrm{i} b}{c} \Delta(\rho, n)\right]\left[\frac{a-\mathrm{i} b}{c}\right]\right\} \exp (-2 \lambda / \pi n) .
\end{aligned}
$$

Hence as $n \rightarrow \infty$, the terms in the products have the following forms in the order in which they appear:

$$
\begin{aligned}
& 1-\frac{\lambda+\mathrm{i} b w}{\pi n}+O\left(\frac{1}{n^{2}}\right), \quad 1-\frac{\lambda-\mathrm{i} b w}{\pi n}+O\left(\frac{1}{n^{2}}\right) \\
& 1+\frac{\lambda-\mathrm{i} b w}{\pi n}+O\left(\frac{1}{n^{2}}\right) \text { and } 1+\frac{\lambda+\mathrm{i} b w}{\pi n}+O\left(\frac{1}{n^{2}}\right) .
\end{aligned}
$$

From this last display we can extract terms which are free of zeros in the lower halfplane $\operatorname{Im} w<0$, that is

$$
\begin{aligned}
& {\left[M_{-}(w)\right]^{2}=c^{2}\left(w-k_{x}\right)\left(w-k_{x}^{*}\right)} \\
& \times \prod_{n=1}^{\infty}\left\{\left[\frac{c w}{\pi n}+\frac{a}{c}\left(1-\frac{k \rho c}{\pi n}\right)-\frac{\mathrm{i} b}{c} \Delta(-\rho, n)\right]\left[\frac{a+\mathrm{i} b}{c}\right]\right\} \exp [(\lambda-\mathrm{i} b w) / \pi n] \\
& \times \prod_{n=1}^{\infty}\left\{\left[-\frac{c w}{\pi n}+\frac{a}{c}\left(1+\frac{k \rho c}{\pi n}\right)+\frac{\mathrm{i} b}{c} \Delta(\rho, n)\right]\left[\frac{a-\mathrm{i} b}{c}\right]\right\} \exp [(-\lambda+\mathrm{i} b w) / \pi n] .
\end{aligned}
$$


Similarly

$$
\begin{aligned}
& {\left[M_{+}(w)\right]^{-2} }=\prod_{n=1}^{\infty}\left\{\left[\frac{c w}{\pi n}+\frac{a}{c}\left(1-\frac{k \rho c}{\pi n}\right)+\frac{\mathrm{i} b}{c} \Delta(-\rho, n)\right]\left[\frac{a-\mathrm{i} b}{c}\right]\right\} \exp [(\lambda+\mathrm{i} b w) / \pi n] \\
& \times \prod_{n=1}^{\infty}\left\{\left[-\frac{c w}{\pi n}+\frac{a}{c}\left(1-\frac{k \rho c}{\pi n}\right)-\frac{\mathrm{i} b}{c} \Delta(-\rho, n)\right]\left[\frac{a+\mathrm{i} b}{c}\right]\right\} \exp [(-i-\mathrm{i} b w) / \pi n]
\end{aligned}
$$

is free of zeros in the smaller of the upper half-planes

$$
\operatorname{Im} w>-\frac{b \pi}{c^{2}} \Delta(-\rho, 1) \text { or }-\frac{b \pi}{c^{2}} \Delta(\rho, 1) .
$$

The asymptotic behaviour of $\left[M_{-}(w)\right]^{2}$ for $|w| \rightarrow \infty, \operatorname{Im} w<0$ is now equivalent to considering the asymptotic behaviour of

Similarly

$$
\begin{aligned}
{\left[M_{-}(w)\right]^{2} \sim } & c^{2} w^{2} \prod_{n=1}^{\infty}\left[1-\frac{\lambda-\mathrm{i} w b}{\pi n}\right] \exp [(\hat{\lambda}-\mathrm{i} w b) / \pi n] \\
& \times \prod_{n=1}^{\infty}\left[1+\frac{\lambda+\mathrm{i} w b}{\pi n}\right] \exp [-(\lambda+\mathrm{i} w b) / \pi n] \\
& \sim \frac{\pi^{2} \exp (-2 \mathrm{i} b w \gamma / \pi)}{\Gamma[(\lambda+\mathrm{i} b w) / \pi] \Gamma[(-\lambda+\mathrm{i} b w) / \pi]}
\end{aligned}
$$

$$
\begin{aligned}
{\left[M_{+}(w)\right]^{-2} \sim } & \prod_{n=1}^{\infty}\left[1-\frac{(\lambda+\mathrm{i} b w)}{\pi n}\right] \exp [(\lambda+\mathrm{i} b w) / \pi n] \\
& \times \prod_{n=1}^{\infty}\left[1+\frac{\lambda-\mathrm{i} b w}{\pi n}\right] \exp [-(\lambda-\mathrm{i} w b) / \pi n]
\end{aligned}
$$

when $|w| \rightarrow \infty$, Im $w>-\varepsilon$, or in terms of the gamma function

$$
\sim \frac{-\pi^{2} \exp [2 \mathrm{i} b w \gamma / \pi]}{c^{2} w^{2} \Gamma[-(\lambda+\mathrm{i} w b) / \pi] \Gamma[(\lambda-\mathrm{i} w b) / \pi]} .
$$

It now follows from the Stirling expansion that

$$
K_{-}(w)=\frac{L_{-}(w)}{M_{-}(w)} \sim w^{1 / 2} \exp \left\{\frac{\mathrm{i} w b}{\pi}\left[2-2 \ln 2-\ln \sin \alpha-\left(\frac{\pi}{2}-\alpha\right) \cot \alpha\right]\right\}
$$

up to a constant factor when $|w| \rightarrow \infty, \operatorname{Im} w<0$. In a similar manner, we find that

$$
\frac{M_{+}(w)}{L_{+}(w)} \sim w^{-1 / 2} \exp \left\{-\frac{i w b}{\pi}\left[2-2 \ln 2-\ln \sin \alpha-\left(\frac{\pi}{2}-\alpha\right) \cot \alpha\right]\right\}
$$

up to a constant factor when $|w| \rightarrow \infty, \operatorname{Im} w>-\varepsilon$. Hence, in order to construct a $K_{-}(w)$ and $K_{+}(w)$ of algebraic order at infinity $\dagger$, we define

$$
K_{-}(w)=\frac{L_{-}(w)}{M_{-}(w)} \exp [\chi(w)]
$$

tRecall that we need this behaviour in order to apply the Liouville theorem referred to in Section 5 . We note that for $\alpha=\pi / 2$, the case which Lüneburg and Hurd ${ }^{6}$ considered, the factor $[\chi(w)]$ does not occur explicitly, but appears implicitly in their discussion. 
and

$$
\frac{1}{K_{+}(w)}=\frac{M_{+}(w)}{L_{+}(w)} \exp [-\chi(w)]
$$

where we have chosen

$$
\chi(w)=-\frac{i w b}{\pi}\left[2-2 \ln 2-\left(\frac{\pi}{2}-\alpha\right) \cot \alpha-\ln \sin \alpha\right]
$$

As we have observed in Section 6, we have need of $\left|K_{+}\left(k_{x}\right)\right|,\left|K_{+}\left(k_{x}^{*}\right)\right|$ and $\left|K_{+}(\kappa)\right|$ in order to determine the magnitudes of the far-field parameters. The determination of $\left|L_{+}\left(k_{x}\right)\right|,\left|L_{+}\left(k_{x}^{*}\right)\right|$ and $\left|L_{+}(\kappa)\right|$ is immediate because $k$ is real. For $w$ real, positive and less than $k$

$$
\begin{aligned}
\left|L_{+}(w)\right|^{-1} & =\left|\prod_{n=1}^{\infty}\left[1-\frac{4 b^{2} \sigma^{2}(w)}{\pi^{2}(2 n+1)^{2}}\right]\right|^{1 / 2} \\
& =\left|\frac{\cos \sigma b}{1-4 b^{2} \sigma^{2} / \pi^{2}}\right|^{1 / 2}
\end{aligned}
$$

Therefore

and

$$
\begin{aligned}
\left|L_{+}\left(k_{x}\right)\right|^{-1} & =\left|\frac{\pi^{2}}{4 b^{2}} \frac{\cos b k_{y}}{\left[\left(k_{x}\right)^{2}-\kappa^{2}\right]}\right|^{1 / 2} \\
\left|L_{+}\left(k_{x}^{*}\right)\right|^{-1} & =\left|\frac{\pi^{2}}{4 b^{2}} \frac{\cos b k_{y}^{*}}{\left[\left(k_{x}^{*}\right)^{2}-\kappa^{2}\right]}\right|^{1 / 2}
\end{aligned}
$$

$$
\left|L_{+}(\kappa)\right|^{-1}=\pi^{1 / 2} / 2
$$

For the determination of $\left|M_{+}\left(k_{x}\right)\right|,\left|M_{+}\left(k_{x}^{*}\right)\right|$ and $\left|M_{+}(\kappa)\right|$ we have that

$$
\begin{aligned}
\left|M_{+}(w)\right|^{-2} & =\mid \prod_{n=1}^{\infty}\left[\left(1-\frac{\lambda}{\pi n}\right)^{2}-\frac{b^{2} \sigma^{2}}{\pi^{2} n^{2}}\right] \exp (2 \lambda / \pi n) \\
& \times\left.\prod_{n=1}^{\infty}\left[\left(1+\frac{\lambda}{\pi n}\right)^{2}-\frac{b^{2} \sigma^{2}}{\pi^{2} n^{2}}\right] \exp (-2 \lambda / \pi n)\right|^{1 / 2} \\
& =\left|\frac{\sin ^{2} \lambda-\sin ^{2} b \sigma}{c^{2}\left(w-k_{x}\right)\left(w-k_{x}^{*}\right)}\right|^{1 / 2}
\end{aligned}
$$

when $-k<w<k$. Hence

and

$$
\begin{aligned}
\left|M_{+}\left(k_{x}\right)\right|^{-2} & =\left|\frac{\sin b k_{y} \cos b k_{y}}{b k_{y}}\right|^{1 / 2} \\
\left|M_{+}\left(k_{x}^{*}\right)\right|^{-2} & =\left|\frac{\sin b k_{y}^{*} \cos b k_{y}^{*}}{b k_{y}^{*}}\right|^{1 / 2}
\end{aligned}
$$

$$
\left|M_{+}(\kappa)\right|^{-2}=\frac{|\cos (k \rho c-a \kappa)|}{\left|c^{2}\left(k_{x}-\kappa\right)\left(k_{x}^{*}-\kappa\right)\right|^{1 / 2}} \text {. }
$$


B. The decomposition of $h(w, c)=g(w) / \sigma(w)$

We are now left with the task of decomposing

$$
h(w, c)=g(w) / \sigma(w)=\frac{1}{2}\left\{\ln \frac{\sin \lambda(w)+\sin b \sigma(w)}{\sin \lambda(w)-\sin b \sigma(w)}\right\} / \sigma(w)
$$

additively, so that one term will be analytic in the smaller of the two upper half-planes $\operatorname{Im} w>-b \pi \Delta(\rho, 1) / c^{2}$ or $-b \pi \Delta(-\rho, 1) / c^{2}$, whereas the other is analytic in the lower half-plane $\operatorname{Im} w<0$. We label the first term $h_{+}(w)$ and the second one $h_{-}(w)$. Each of these terms has two representations, one of which is valid when $|w|<k$ and the other when $|w|>k$. The representation which is valid when $|w|<k$ is needed to calculate the far-field parameters, whereas the one which is valid for $|w|>k$ is needed to determine the necessary asymptotic forms which are required to find the entire functions $E_{1}(w)$ and $E_{2}(w)$, [see Section V]. With the branch of the logarithm in (B1) chosen to be zero when $w=k$ and $\sigma(w)$ to be real and positive when $w$ is real and $-k<w<k, h(w, c)$ is a single-valued analytic function in the smaller of the strips $-b \pi \Delta(\rho, 1) / c^{2}<\operatorname{Im} w<0$ or $-\pi b \Delta(-\rho, 1) / c^{2}<\operatorname{Im} w<0$. An elementary calculation enables us to write (B1) additively as a term which is immediately analytic in the lower half-plane $\operatorname{Im} w<0$ and one which is analytic in the smaller of the strips just described. An application of the Cauchy integral theorem to this last term will complete the decomposition we seek.

To this end we write

$$
h(w, c)=\frac{1}{2}\left\{\ln \frac{\sin (\lambda+b \sigma) / 2 \cos (\lambda-b \sigma) / 2}{\sin (\lambda-b \sigma) / 2 \cos (\lambda+b \sigma) / 2}\right\} / \sigma(w)
$$

and upon noting the product representation of the various trigonometric functions, we obtain

$$
\begin{aligned}
h(w, c)= & \frac{1}{2 \sigma(w)}\left\{\ln \frac{\lambda+b \sigma}{\lambda-b \sigma} \prod_{n=1}^{\infty} \frac{1-\left(\frac{\lambda+b \sigma}{2 n \pi}\right)^{2}}{1-\left(\frac{\lambda-b \sigma}{2 n \pi}\right)^{2}}\right. \\
& \left.\times \prod_{n=1}^{\infty} \frac{1-\left[\frac{\lambda-b \sigma}{(2 n-1) \pi}\right]^{2}}{1-\left[\frac{\lambda+b \sigma}{(2 n-1) \pi}\right)^{2}}\right\}(\sigma(w) \\
= & \frac{1}{2 \sigma(w)}\left\{\ln \frac{\lambda+b \sigma}{\lambda-b \sigma} \prod_{n=1}^{\infty}\left[\frac{1+\frac{\lambda+b \sigma}{2 n \pi}}{1+\frac{b \sigma-\lambda}{2 n \pi}}\right]^{2}\right. \\
& \times \prod_{n=1}^{\infty} \frac{[\lambda / n \pi}{\left[1+\frac{\lambda}{2 n \pi}\right]^{2}-\frac{b^{2} \sigma^{2}}{4 n^{2} \pi^{2}}} \mathrm{e}^{2 \lambda / n \pi} \\
& {\left[1-\frac{b^{2} \sigma^{2}}{4 n^{2} \pi^{2}}\right.}
\end{aligned}
$$




$$
\begin{aligned}
& \times \prod_{n=1}^{\infty}\left[\frac{1+\frac{b \sigma-\lambda}{(2 n-1) \pi}}{1+\frac{b \sigma+\lambda}{(2 n-1) \pi}}\right]^{2} \mathrm{e}^{4 \lambda /(2 n-1) \pi} \\
& \left.\times \prod_{n=1}^{\infty} \frac{\left[1+\frac{\lambda}{(2 n-1) \pi}\right]^{2}-\frac{b^{2} \sigma^{2}}{(2 n-1)^{2} \pi^{2}}}{\left[1-\frac{\lambda}{(2 n-1) \pi}\right]^{2}-\frac{b^{2} \sigma^{2}}{(2 n-1)^{2} \pi^{2}}} \mathrm{e}^{-4 \lambda /(2 n-1) \pi}\right\} .
\end{aligned}
$$

The first term inside the braces and the first and third products are analytic in the lower half-plane $\operatorname{Im} w<0$. For later use we note that (B2) can be rewritten as

$$
\begin{aligned}
h(w, c)= & \frac{1}{2 \sigma(w)}\left\{\ln \frac{\lambda+b \sigma}{\lambda-b \sigma}\left[\prod_{n=1}^{\infty} \frac{1+\frac{b \sigma+\lambda}{2 n \pi}}{1+\frac{b \sigma-\lambda}{2 n \pi}} \mathrm{e}^{-\lambda / n \pi}\right]^{4}\right. \\
& \times\left[\prod_{n=1}^{\infty} \frac{1+\frac{b \sigma-\lambda}{n \pi}}{1+\frac{b \sigma+\lambda}{n \pi}} \mathrm{e}^{2 \lambda / n \pi}\right]^{2}\left[\prod_{n=1}^{\infty} \frac{\left(1-\frac{\lambda}{2 n \pi}\right)^{2}-\frac{b^{2} \sigma^{2}}{4 n^{2} \pi^{2}}}{\left(1+\frac{\lambda}{2 n \pi}\right)^{2}-\frac{b^{2} \sigma^{2}}{4 n^{2} \pi^{2}}} \mathrm{e}^{2 \lambda / n \pi}\right]^{2} \\
& \left.\times\left[\prod_{n=1}^{\infty} \frac{\left(1+\frac{\lambda}{n \pi}\right)^{2}-\frac{b^{2} \sigma^{2}}{n^{2} \pi^{2}}}{\left(1-\frac{\lambda}{n \pi}\right)^{2}-\frac{b^{2} \sigma^{2}}{n^{2} \pi^{2}}} \mathrm{e}^{-4 \lambda / n \pi}\right]\right\}
\end{aligned}
$$

Now we observe that the third and fourth products in (B3) have zeros and poles in the upper and lower half-planes. The factor $(1-\hat{\lambda} / n \pi)^{2}-b^{2} \sigma^{2} / n^{2} \pi^{2}, n=1,2, \ldots$ has zeros in the lower half-plane at $w_{n}^{-}=[-a(n \pi-k \rho c)-\mathrm{i} b n \pi \Delta(-\rho, n)] / c^{2}$ where $\Delta(-\rho, n)=\left[(1-k \rho c / n \pi)^{2}-c^{2} k^{2} / n^{2} \pi^{2}\right]^{1 / 2}>0$, while the factor $(1+\lambda / n \pi)^{2}-b^{2} \sigma^{2} / n^{2} \pi^{2}$ has zeros in the lower half-plane at $w_{n}^{+}=[a(n \pi+k \rho c)-i b n \pi \Delta(\rho, n)] / c^{2}$. The zeros of these factors in the upper half-plane are at $\bar{w}_{n}^{ \pm}$, the complex conjugate of $w_{n}^{ \pm}$ and $\Delta(\rho, n)>0$. We shall ultimately need $\sigma\left(w_{n}^{ \pm}\right)$which may be calculated from $1-\lambda\left(w_{n}^{ \pm}\right) / n \pi=b \sigma\left(w_{n}^{ \pm}\right) / n \pi$. These $\sigma$ s are

and

$$
\sigma\left(w_{n}^{-}\right)=[b(n \pi-k \rho c)-\mathrm{i} a n \pi \Delta(-\rho, n)] / c^{2}
$$

$$
\sigma\left(w_{n}^{+}\right)=[b(n \pi+k \rho c)+\mathrm{i} a n \pi \Delta(\rho, n)] / c^{2} .
$$

The last two products in (B3) can therefore be written in terms of $w_{n}^{ \pm}$and $\bar{w}_{n}^{ \pm}$as

$$
\begin{aligned}
& \left\{\prod_{n=1}^{\infty} \frac{\left[(-a+\mathrm{i} b)\left(w_{2 n}^{-}-w\right) / 2 n \pi\right]\left[(-a-\mathrm{i} b)\left(\bar{w}_{2 n}^{-}-w\right) / 2 n \pi\right]}{\left[(a+\mathrm{i} b)\left(w_{2 n}^{+}-w\right) / 2 n \pi\right]\left[(a-\mathrm{i} b)\left(\bar{w}_{2 n}^{+}-w\right) / 2 n \pi\right]} \mathrm{e}^{2 \lambda / n \pi}\right\}^{2} \\
& \times \sum_{n=1}^{\infty} \frac{\left[(a+\mathrm{i} b)\left(w_{n}^{+}-w\right) / n \pi\right]\left[(a-\mathrm{i} b)\left(\bar{w}_{n}^{+}-w\right) / n \pi\right]}{\left[(-a+\mathrm{i} b)\left(w_{n}^{-}-w\right) / n \pi\right]\left[(-a-\mathrm{i} b)\left(\bar{w}_{n}^{-}-w\right) / n \pi\right]} \mathrm{e}^{-4 \lambda / n \pi} .
\end{aligned}
$$

The role of the expressions $(a \pm i b) / c^{2}$ is to arrange the factors in the product so that 
when $n$ is sufficiently large, the terms will be $1+O(1 / n)$. Now (B4) can be rewritten as

$$
\begin{aligned}
& \prod_{n=1}^{\infty}\left[\frac{(-a+\mathrm{i} b)\left(w_{2 n}^{-}-w\right) / 2 n \pi}{(a+\mathrm{i} b)\left(w_{2 n}^{+}-w\right) / 2 n \pi}\right]^{2}\left[\frac{(a+\mathrm{i} b)\left(w_{n}^{+}-w\right) / n \pi}{(-a+\mathrm{i} b)\left(w_{n}^{-}-w\right) / n \pi}\right] \\
& \times \prod_{n=1}^{\infty}\left[\frac{(-a-\mathrm{i} b)\left(\bar{w}_{2 n}^{-}-w\right) / 2 n \pi}{(a-\mathrm{i} b)\left(\bar{w}_{2 n}^{+}-w\right) / 2 n \pi}\right]^{2}\left[\frac{(a-\mathrm{i} b)\left(\bar{w}_{n}^{+}-w\right) / n \pi}{(-a-\mathrm{i} b)\left(\bar{w}_{n}^{-}-w\right) / n \pi}\right] .
\end{aligned}
$$

Observe that there are no convergence factors in the last display. Of these two products, the first one is free of zeros and poles in the upper half-plane $\operatorname{Im} w>-\varepsilon_{1}$, whereas the second one is free of zeros and poles in the lower half-plane $\operatorname{Im} w<-\varepsilon_{2}$. We have then that $\{\ln (\sin \lambda+\sin b \sigma) /(\sin \lambda-\sin b \sigma)\} / \sigma(w)$ is composed of four products and the term $(\lambda+b \sigma) /(\lambda-b \sigma)$. Only the term

$$
\left\{\ln \prod_{n=1}^{\infty}\left[\frac{(-a+\mathrm{i} b)\left(w_{2 n}^{-}-w\right) / 2 n \pi}{(a+\mathrm{i} b)\left(w_{2 n}^{+}-w\right) / 2 n \pi}\right]^{2}\left[\frac{(a+\mathrm{i} b)\left(w_{n}^{+}-w\right) / n \pi}{(-a+\mathrm{i} b)\left(w_{n}^{-}-w\right) / n \pi}\right]\right\} / \sigma(w)
$$

is analytic in the strip $-\varepsilon_{1}<\operatorname{Im} w<-\varepsilon_{2}$ owing to the branch points of $\sigma(w)$, whereas the others are analytic in the lower half-plane $\operatorname{Im} w<-\varepsilon_{2}$.

We can therefore determine $h_{+}(w, c)$, the part of $h(w, c)$ which is analytic in the upper half-plane $\operatorname{Im} w>-\varepsilon_{1}$ by evaluating the Cauchy integral

$$
\frac{1}{2 \pi \mathrm{i}} \int \frac{h(t, c) \mathrm{d} t}{t-w}
$$

along a rectangle whose sides are straight lines which run from $-L-\mathrm{i} \varepsilon_{2}$ to $L-\mathrm{i} \varepsilon_{2}$, $L-\mathrm{i} \varepsilon_{2}$ to $L-\mathrm{i} \varepsilon_{1} L-\mathrm{i} \varepsilon_{1}$ to $-L-\mathrm{i} \varepsilon_{1}$ and $-L-\mathrm{i} \varepsilon_{1}$ to $-L-\mathrm{i} \varepsilon_{2}$. In the limit $L \rightarrow \infty$, the integrals along the vertical segments make no contributions. The integral

$$
\frac{1}{2 \pi \mathrm{i}} \int_{-\infty-\mathrm{i} \varepsilon_{1}}^{\infty-\mathrm{i} \varepsilon_{1}} \frac{h(t, c) \mathrm{d} t}{t-w}=h_{+}(w, c)
$$

provides the term which is analytic in the upper half-plane $\operatorname{Im} w>-\varepsilon_{1}$ by analytic continuation. The integral

$$
\frac{1}{2 \pi \mathrm{i}} \int_{\infty-\mathrm{i} \varepsilon_{2}}^{-\infty-\mathrm{i} \varepsilon_{2}} \frac{h(t, c) \mathrm{d} t}{t-w}=h_{-}(w, c)
$$

now determines the term which is analytic in the lower half-plane.

The integrand in (B6) has logarithmic branch point singularities in the lower halfplane $\operatorname{Im} w<-\varepsilon_{2}$ at $w_{n}^{ \pm}, n=1,2, \ldots$ This integral may be evaluated by first drawing branch cuts from $w_{n}^{ \pm}$to $\infty \exp \left[i \arg w_{n}^{ \pm}\right]$. We then choose a line segment which runs from $-R-\mathrm{i} \varepsilon_{1}$ to $R-\mathrm{i} \varepsilon_{1}$ and a semicircular path of radius $R$ in the lower half-plane, of centre $\left(0,-\varepsilon_{1}\right)$ which is deformed to pass about these branch cuts for each $n$. By Cauchy's theorem, this integral vanishes, and when $R \rightarrow \infty$ there are no contributions from the integral along the circular arcs. We are then left with the integrals along the cuts which arise from the change of phase of $h(t, c)$ along the cuts. After this operation is completed, we have that (B6) reduces to

$$
\begin{aligned}
& \sum_{n=1}^{\infty}\left[2 \int_{\infty \exp \left[\operatorname{iarg} w_{2 n}^{-}\right]}^{w_{2 n}^{-}}-\int_{\infty \exp \left[\operatorname{iarg} w_{n}^{-}\right]}^{w_{n}^{-}}+\int_{\infty \exp \left[\operatorname{iarg} w_{n}^{+}\right]}^{w_{n}^{+}}\right] \frac{\mathrm{d} t}{2 \sigma(t)(t-w)} .
\end{aligned}
$$


This in turn may be simplified to

$$
\frac{1}{2} \sum_{n=1}^{\infty}\left[2 \int_{w_{2 n}^{+}}^{w_{2 n}^{-}}+\int_{w_{n}^{-}}^{w_{n}^{+}}\right] \frac{\mathrm{d} t}{\sigma(t)(t-w)}
$$

and this reduces to

$$
\begin{aligned}
& \frac{1}{2 \sigma(w)} \sum_{n=1}^{\infty}\left\{\operatorname { l n } \left[\frac{\sqrt{ }(k-w) \sqrt{ }\left(k+w_{2 n}^{+}\right)+\sqrt{ }(k+w) \sqrt{ }\left(k-w_{2 n}^{+}\right)}{\sqrt{ }(k-w) \sqrt{ }\left(k+w_{2 n}^{+}\right)-\sqrt{ }(k+w) \sqrt{ }\left(k-w_{2 n}^{+}\right)}\right.\right.
\end{aligned}
$$

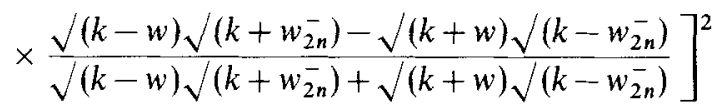

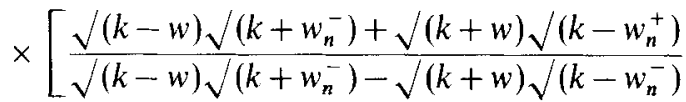

$$
\begin{aligned}
& \left.\left.\times \frac{\sqrt{ }(k-w) \sqrt{ }\left(k+w_{n}^{+}\right)-\sqrt{ }(k+w) \sqrt{ }\left(k-w_{n}^{+}\right)}{\sqrt{ }(k-w) \sqrt{ }\left(k+w_{n}^{+}\right)+\sqrt{ }(k+w) \sqrt{ }\left(k-w_{n}^{+}\right)}\right]\right\} .
\end{aligned}
$$

There are two alternative forms for (B9) which are useful. The first one is obtained by squaring the argument of the logarithm and dividing the logarithm by 2 . In this case we obtain

$$
\begin{aligned}
h_{+}(w, c)= & \frac{1}{4 \sigma(w)}\left\{\ln \prod_{n=1}^{\infty}\left[\frac{k^{2}-w w_{2 n}^{+}+\sigma(w) \sigma\left(w_{2 n}^{+}\right)}{k^{2}-w w_{2 n}^{+}-\sigma(w) \sigma\left(w_{2 n}^{+}\right)} \frac{k^{2}-w w_{2 n}^{-}-\sigma(w) \sigma\left(w_{2 n}^{-}\right)}{k^{2}-w w_{2 n}^{-}+\sigma(w) \sigma\left(w_{2 n}^{-}\right)}\right]^{2}\right. \\
& \left.\times\left[\frac{k^{2}-w w_{n}^{-}+\sigma(w) \sigma\left(w_{n}^{-}\right) k^{2}-w w_{n}^{+}-\sigma(w) \sigma\left(w_{n}^{+}\right)}{k^{2}-w w_{n}^{-}-\sigma(w) \sigma\left(w_{n}^{-}\right)} \frac{k^{2}-w w_{n}^{+}+\sigma(w) \sigma\left(w_{n}^{+}\right)}{k^{-}}\right]\right\} .
\end{aligned}
$$

The second form is obtained from the last one upon noting that $\left[k^{2}-w w_{n}^{ \pm}\right.$ $\left.+\sigma(w) \sigma\left(w_{n}^{ \pm}\right)\right]\left[k^{2}-w w_{n}^{ \pm}-\sigma(w) \sigma\left(w_{n}^{ \pm}\right)\right]=k^{2}\left(w-w_{n}^{ \pm}\right)^{2}$. Here we obtain

$$
\begin{aligned}
h_{+}(w, c)= & \frac{1}{2 \sigma(w)} \ln \prod_{n=1}^{\infty}\left\{\left[\frac{k^{2}-w w_{2 n}^{+}+\sigma(w) \sigma\left(w_{2 n}^{+}\right)}{k^{2}-w w_{2 n}^{-}+\sigma(w) \sigma\left(w_{2 n}^{-}\right)} \frac{\left(w-w_{2 n}^{-}\right)}{\left(w-w_{2 n}^{+}\right)}\right]^{2}\right. \\
& \times\left[\frac{k^{2}-w w_{n}^{-}+\sigma(w) \sigma\left(w_{n}^{-}\right)}{k^{2}-w w_{n}^{+}+\sigma(w) \sigma\left(w_{n}^{+}\right)\left(w-w_{n}^{+}\right)} \frac{(w)}{\left(w-w_{n}^{-}\right)}\right\}=\frac{g_{+}(w)}{\sigma(w)}
\end{aligned}
$$

(B11) displays the branch points in the lower half-plane explicitly.

We now show that $h_{+}(w, c)$ as given by $(\mathrm{B} 10)$ is a single-valued function in the smaller of the upper half-plane $\operatorname{Im} w>-\pi b \Delta(\rho, 1) / c^{2}$ or $-\pi b \Delta(-\rho, 1) / c^{2}$. In order to see this, we note the only possible singularities of $h_{+}(w, c)$ in these half-planes are the branch points at $w= \pm k$. We draw straight line branch cuts from $-\infty$ to $-k$ and $k$ to $\infty$. Then $\sigma(w)=\mathrm{i}\left(w^{2}-k^{2}\right)^{1 / 2}$ on the lower side of the cut from $k$ to $\infty$ if we take $\sigma(w)>0$ on $-k<w<k$. On the other hand $\sigma(w)=-\mathrm{i}\left(w^{2}-k^{2}\right)^{1 / 2}$ on the upper side of this cut. Hence, since there are no zeros in the products in (B10) in the upper halfplane, $h_{+}(w, c)$ remains unchanged. The same remarks may be made about the cut from $-\infty$ to $-k$.

Clearly $h_{+}(w, c)$ has only branch points at $w_{n}^{ \pm}, n=1,2, \ldots$ which lie in the lower half-plane $\operatorname{lm} w<-\varepsilon_{1}$. It is also clear that the infinite product converges and is an analytic function in the upper half-plane which we described since it is an alternative representation of the integral which defines $h_{+}(w, c)$ in that half-plane. In order to 
verify directly that the product in (B10) does not require convergence factors we divide all factors in the product containing the index $n$ by $n \pi$ and those containing the index $2 n$ by $2 n \pi$. Then for $n$ sufficiently large, we have for a $w_{n}^{+}$term

$$
\begin{aligned}
& \frac{k^{2} / \pi n-(w-\mathrm{i} \sigma)(a-\mathrm{i} b)(1+k \rho c / \pi n) / c^{2}}{k^{2} / \pi n-(w+\mathrm{i} \sigma)(a-\mathrm{i} b)(1+k \rho c / \pi n) / c^{2}} \\
& =\frac{w-\mathrm{i} \sigma}{w+\mathrm{i} \sigma} \frac{1+[k \rho c-(a+\mathrm{i} b)(w+\mathrm{i} \sigma)] / \pi n}{1+[k \rho c-(a+\mathrm{i} b)(w-\mathrm{i} \sigma)] / \pi n}
\end{aligned}
$$

A product with such a term requires a convergence factor $\exp [2 \mathrm{i} \sigma(a+\mathrm{i} b) / n \pi]$. On the other hand for $n$ sufficiently large, we have for a $w_{n}^{-}$term

$$
\frac{w+\mathrm{i} \sigma}{w-\mathrm{i} \sigma} \frac{1+[(a-\mathrm{i} b)(w-\mathrm{i} \sigma)-k \rho c] / \pi n}{1+[(a-\mathrm{i} b)(w+\mathrm{i} \sigma)-k \rho c] / \pi n} .
$$

This term now requires a convergence factor $\exp [2 \mathrm{i} \sigma(a-\mathrm{i} b) / \pi n]$. Hence for the product with index $n$, we only require a convergence factor $\exp [4 i a \sigma / \pi n]$ and the external terms $(w-\mathrm{i} \sigma) /(w+\mathrm{i} \sigma)$ and $(w+\mathrm{i} \sigma) /(w-\mathrm{i} \sigma)$ cancel. For the terms containing the index $2 n$, we require a convergence factor $\exp [-2 \mathrm{i} a \sigma / \pi n]$ and this in turn has to be squared. Hence no convergence factor is required in (B10).

In order to calculate $h_{-}(w, c)$ we write

$$
h_{-}(w, c)=\frac{1}{2 \sigma(w)} \ln \frac{\sin \lambda+\sin b \sigma}{\sin \lambda-\sin b \sigma}-h_{+}(w, c)
$$

rather than attempting to evaluate the integral for $h_{-}(w, c)$. The following identities will be useful in simplifying the right side of (B12). We have

$$
\begin{aligned}
& {\left[k^{2}-w w_{n}^{-}+\sigma(w) \sigma\left(w_{n}^{-}\right)\right]\left[k^{2}-w \bar{w}_{n}^{-}+\sigma(w) \sigma\left(\bar{w}_{n}^{-}\right)\right]} \\
& =k^{2} n^{2} \pi^{2}[1+(b \sigma-\lambda) / n \pi]^{2} / c^{2}, \\
& {\left[k^{2}-w w_{n}^{+}+\sigma(w) \sigma\left(w_{n}^{+}\right)\right]\left[k^{2}-w \bar{w}_{n}^{+}+\sigma(w) \sigma\left(\bar{w}_{n}^{+}\right)\right]} \\
& =k^{2} n^{2} \pi^{2}[1+(b \sigma+\lambda) / n \pi]^{2} / c^{2}, \\
& \left(1-\frac{\lambda}{n \pi}\right)^{2}-\frac{b^{2} \sigma^{2}}{n^{2} \pi^{2}}=c^{2}\left(w-w_{n}^{-}\right)\left(w-\bar{w}_{n}^{-}\right) / n^{2} \pi^{2}
\end{aligned}
$$

and

$$
\left(1+\frac{\lambda}{n \pi}\right)^{2}-\frac{b^{2} \sigma^{2}}{n^{2} \pi^{2}}=c^{2}\left(w-w_{n}^{+}\right)\left(w-\bar{w}_{n}^{+}\right) / n^{2} \pi^{2} .
$$

Upon simplifying (B12) with the aid of these identities, we obtain

$$
\begin{aligned}
h_{-}(w, c)= & \frac{1}{2 \sigma(w)} \ln \frac{\lambda+b \sigma}{\lambda-b \sigma} \prod_{n=1}^{\infty}\left\{\begin{array}{l}
k^{2}-w \bar{w}_{n}^{+}-\sigma(w) \sigma\left(\bar{w}_{n}^{+}\right) \\
k^{2}-w \bar{w}_{n}^{+}+\sigma(w) \sigma\left(\bar{w}_{n}^{+}\right)
\end{array}\right. \\
& \left.\times \frac{k^{2}-w \bar{w}_{n}^{-}+\sigma(w) \sigma\left(\bar{w}_{n}^{-}\right)}{k^{2}-w \bar{w}_{n}^{-}-\sigma(w) \sigma\left(\bar{w}_{n}^{-}\right)}\right\} \\
& \times\left\{\frac{k^{2}-w \bar{w}_{2 n}^{+}+\sigma(w) \sigma\left(\bar{w}_{2 n}^{+}\right)}{k^{2}-w \bar{w}_{2 n}^{+}-\sigma(w) \sigma\left(\bar{w}_{2 n}^{+}\right)} \frac{k^{2}-w \bar{w}_{2 n}^{-}-\sigma(w) \sigma\left(\bar{w}_{2 n}^{-}\right)}{k^{2}-w \bar{w}_{2 n}^{-}+\sigma(w) \sigma\left(\bar{w}_{2 n}^{-}\right)}\right\}^{2}=\frac{g_{-}(w)}{\sigma(w)}
\end{aligned}
$$


and $h_{-}(w, c)$ is analytic in the lower half-plane $\operatorname{Im} w<0$. There is an alternative form of (B13) which displays the branch points of $h_{-}(w, c)$ at $\bar{w}_{n}^{-}$and $\bar{w}_{n}^{+}, n=1,2, \ldots$

We can now determine $\mid \exp \left[g_{+}(w) \mid\right.$, where $g_{+}(w)=\sigma(w) h_{+}(w, c)$ for $-k<w<k$ following the method which we employed in Section A. We have here

$$
\mid \exp \left[g_{+}(w)|=|[\lambda-b \sigma][\cot (\hat{\lambda}-b \sigma) / 2][\tan (\hat{\lambda}+b \sigma) / 2] /\left.[\lambda+b \sigma]\right|^{1 / 4}\right.
$$

and therefore

and

$$
\begin{aligned}
& \left|\exp g_{+}\left(k_{x}\right)\right|=\left|\tan b k_{y} / 2 b k_{y}\right|^{1 / 4}, \\
& \left|\exp g_{+}\left(k_{x}^{*}\right)\right|=\left|\tan b k_{y}^{*} / 2 b k_{y}^{*}\right|^{1 / 4}
\end{aligned}
$$

$$
\left|\exp g_{+}(\kappa)\right|=\left[\frac{1+\sin \lambda(\kappa)}{1-\sin \lambda(\kappa)} \frac{\pi / 2-\lambda(\kappa)}{\pi / 2+\lambda(\kappa)}\right]^{1 / 4}
$$

\section{The asymptotic behaviour of $g_{+}(w)$ and $g_{-}(w)$}

In order to apply the ideas of Wiener and Hopf in the present case, it is essential that $g_{+}(w)$ and $g_{-}(w)$ be bounded at infinity in their respective half-planes of analyticity (see Section 5, where these facts are needed). We shall find that $g_{+}(w)$ and $g_{-}(w)$ are bounded at infinity in their respective half-planes of analyticity and therefore so are $\cosh \left[g_{+}(w)\right], \sinh \left[g_{+}(w)\right], \cosh \left[g_{-}(w]\right.$ and $\sinh \left[g_{-}(w)\right]$ at infinity in those halfplanes.

The integral (B8) has been evaluated on the premise that $|w|<k$. We now need a form which is useful when $|w|>k$. In this case (B8) takes the form

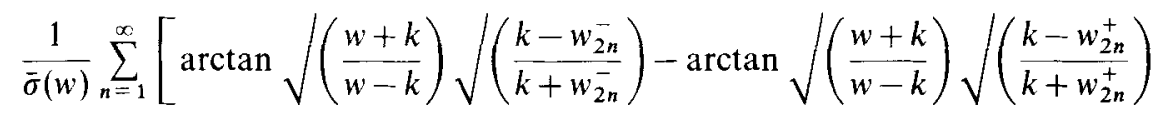

$$
\begin{aligned}
& \left.+\arctan \sqrt{ }\left(\frac{w+k}{w-k}\right) \sqrt{ }\left(\frac{k-w_{2 n-1}^{+}}{k+w_{2 n-1}^{+}}\right)-\arctan \sqrt{ }\left(\frac{w+k}{w-k}\right) \sqrt{ }\left(\frac{k-w_{2 n-1}^{-}}{k+w_{2 n-1}^{-}}\right)\right] \text {, }
\end{aligned}
$$

where $\bar{\sigma}(w)=\left(w^{2}-k^{2}\right)^{1 / 2}$ and is positive when $\operatorname{Re} w>k, \operatorname{Im} w=0$. (C1) in turn, may be rewritten with the aid of the double angle formula for the tangent function as

$$
\begin{aligned}
& \frac{1}{2 \bar{\sigma}(w)} \sum_{n=1}^{\infty}\left[\arctan \frac{\bar{\sigma}(w) \sigma\left(w_{2 n}^{-}\right)}{w w_{2 n}^{-}-k^{2}}-\arctan \frac{\bar{\sigma}(w) \sigma\left(w_{2 n}^{+}\right)}{w w_{2 n}^{+}-k^{2}}\right. \\
& \left.+\arctan \frac{\bar{\sigma}(w) \sigma\left(w_{2 n-1}^{+}\right)}{w w_{2 n-1}^{+}-k^{2}}-\arctan \frac{\bar{\sigma}(w) \sigma\left(w_{2 n-1}^{-}\right)}{w w_{2 n-1}^{-}-k^{2}}\right]=\frac{g_{+}(w)}{\bar{\sigma}(w)} .
\end{aligned}
$$

This last expression shows that there are no branch points at $w= \pm k$ for the function $g_{+}(w) / \bar{\sigma}(w)$. On the other hand when $|w| \rightarrow \infty, \operatorname{Im} w>-\varepsilon_{1}, g_{+}(w)$ reduces to

$$
\sum_{n=1}^{\infty}\left[\arctan \frac{\sigma\left(w_{2 n}^{-}\right)}{w_{2 n}^{-}}-\arctan \frac{\sigma\left(w_{2 n}^{+}\right)}{w_{2 n}^{+}}+\arctan \frac{\sigma\left(w_{2 n-1}^{+}\right)}{w_{2 n-1}^{+}}-\arctan \frac{\sigma\left(w_{2 n-1}^{-}\right)}{w_{2 n-1}^{-}}\right] .
$$

Upon examining the expressions which we found for $w_{n}^{ \pm}$and $\sigma\left(w_{n}^{ \pm}\right)$when $n$ is sufficiently large, we observe that the terms in the bracket are $O\left(1 / n^{2}\right)$ and therefore the series converges. Hence $g_{+}(w)$ is bounded when $|w| \rightarrow \infty, \operatorname{Im} w>-\varepsilon_{1}$. A similar remark is available for $g_{-}(w)$ in the lower half-plane. 


\section{References}

1. Carlson, J. F. and Heins, A. E., 'The reflection of an electromagnetic plane wave by an infinite set of plates I', Quart. Appl. Math., 4, 313-329 (1947); Heins, A. E. and Carlson, J. F., 'The reflection of an electromagnetic plane wave by an infinite set of plates II', Quart. Appl. Math., 5, 82-88 (1947).

2. Daniele, V. G., 'On the factorization of Wiener-Hopf matrices in problems solvable with Hurd's method', IEEE Trans. on Antennas and Propagation, AP-26, 614-616 (1978).

3. Heins, A. E., 'The Green's function for periodic structures in diffraction theory with an application to parallel plate media, I and II', J. Math. Mech., 6, 401-426; 629-639 (1957).

4. Heins, A. E., 'Systems of Wiener-Hopf integral equations and their applications to some boundary value problems in electromagnetic theory', Proc. of Symposium in Appl. Math. Vol. II, 1950, pp. 76-81.

5. Korevaar, J., Mathematical Methods, Vol. I, Academic Press, New York; London, 1968.

6. Lüneburg, E. and Hurd, R. A., 'Diffraction by an infinite set of hard and soft parallel half-planes', Canad. J. Phys., 60, 1-9 (1982).

7. Meister, E., 'Zum Dirichlet-Problem der Helmholtzschen Schwingungsgleichung für ein gestaffeltes Streckengitter., Archive for Rational Mechanics and Analysis, 10, 67-100 (1962). 